\title{
SYNTHESIS, CHARACTERIZATION AND COMPARATIVE STUDY OF \\ SOME DIOXOMOLYBDENUM(VI) SCHIFF BASES COMPLEXES \\ DERIVED FROM AROYL HYDRAZONE
}

\author{
M.A Katkara, S.N. Rao and H.D. Juneja ${ }^{b}$ \\ aDepartment of Chemistry, Priyadarshini Institute of Engg, and \\ Technology, Hingna road, Nagpur-440019, India. \\ bDept. of Chemistry, R.T.M. Nagpur University, Nagpur-440010, India. \\ Corresponding author E-mail : mrinalkatkarmak@yahoo.co.in \\ Tel- 07104-236463; Fax-07104-4236458
}

\begin{abstract}
Synthesis and characterization and comparative study of some novel dioxomolybdenum (VI) complexes having the general formula cis-[ $\left.\mathrm{MoO}_{2}(\mathrm{~L})(\mathrm{solv})\right]$ (where $\mathrm{LH}_{2}=$ Schiff base derived from nicotinic acid hydrazide(NAH), benzoic acid hydrazide(BAH), salicylic acid hydrazide(SAH) and o-hydroxyacetophenone(hap) and salicyladehyde (sal) and solv=ethanol $/ \mathrm{H}_{2} \mathrm{O}$ ) are reported. The various Schiff base ligands are prepared by condensation of nicotinic acid hydrazide(NAH), benzoic acid hydrazide(BAH), salicylic acid hydrazide (SAH) with salicylaldehyde and substituted salicylaldehyde The cis-[ $\left.\mathrm{MoO}_{2}(\mathrm{~L})(\mathrm{solv})\right]$ complexes are synthesized by reacting cis$\left[\mathrm{MoO}_{2}(\mathrm{acac})_{2}\right]$ and the ligands in ethanol according to the reaction,

$\Delta, 6 \mathrm{hrs}$

cis $-\mathrm{MoO}_{2}(\text { acac })_{2}+\mathrm{LH}_{2}$ cis $-\left[\mathrm{MoO}_{2}(\mathrm{~L})(\mathrm{S})\right]+2 \mathrm{acacH}$

$\mathrm{EtOH}$

Where $\mathrm{S}=$ Solvent.

These Schiff base ligands and their $\mathrm{MoO}_{2}$ complexes are characterized by elemental analysis, IR, NMR, UV-Vis and conductivity measurements, thermo gravimetric and powder X-ray diffraction analysis. The spectral studies suggest that the Schiff base ligands behave as dibasic tridentate ligands and co-ordinate through phenolic oxygen, azomethine nitrogen and enolic oxygen atoms. The complexes are found to be monomers, non-electrolytes, diamagnetic, six-coordinated, and crystalline and adopt triclinic crystal system with $\mathrm{P}$ type lattice. The sixth coordination site is occupied by a labile solvent molecule.
\end{abstract}

KEYWORDS: Schiff bases, hydrazones, Dioxomolybdenum complexes.

\section{INTRODUCTION}

Molybdenum complexes with Schiff base ligands have gained considerable importance due to their potential for biological and 
physiological activity and industrial use [1-6]. Studies on complexes of oxomolybdenum(V) and dioxomolybdenum(VI) have opened up a new vista of research and analysis of biochemical significance. A great deal of work has been carried out in the synthesis and characterization of oxo-, dioxo-molybdenum complexes with one vacant site which might allow binding and displacement of various substrates [7]. The availability of such a labile site imparts catalytic property to these complexes. Dibasic tridentate Schiff base ligands around octahedral molybdenum (VI) provide suitable geometry with one vacant site for substrate binding. We have been interested in five co-ordinated octahedral cis- $\mathrm{MoO}_{2}$ complexes with non-symmetrical Schiff base having mixed sets of donor atoms in which the sixth co-ordination site is occupied by a solvent molecule. In view of this, we report the synthesis, structural characterization and comparative study of some cis-dioxomolybdenum(VI) complexes with Schiff bases derived from salicylaldehyde(sal) and ohydroxybenzophenone with nicotinic acid hydrazide(NAH), benzoic acid hydrazide(BAH) and salicylic acid hydrazide(SAH). These Schiff bases form mononuclear dioxomolybdenum(VI) complexes having the general formula $\mathrm{MoO}_{2}(\mathrm{~L})(\mathrm{S})$ (where $\mathrm{LH}_{2}=$ Schiff base represented as, $\mathrm{H}_{2}$ sal-NAH, $\mathrm{H}_{2}$ sal -BAH, $\mathrm{H}_{2}$ sal -SAH, $\mathrm{H}_{2}$ hbp-NAH, $\mathrm{H}_{2}$ hbp -BAH, $\mathrm{H}_{2}$ hbp -SAH). The ligands and the complexes are characterized by elemental analysis, molar conductance and spectroscopic (IR, ${ }^{1} \mathrm{HNMR}$ and UV-Vis), thermogravimetric (TGA) and X-ray diffraction analysis.

\section{EXPERIMENTAL}

\section{MATERIALS}

Ammonium molybdate(VI) tetrahydrate was obtained from Sisco Research Laboratory, (Mumbai, India). Salicylaldehyde, ohydroxyacetophenone,o-hydroxypropiophenone, o-hydroxybenzophenone and benzoic acid hydrazide were procured from Lancaster synthesis Ltd. (UK). Ethanol and acetone used as solvent for synthesis were of high purity. $\mathrm{MoO}_{2}$ (acac) 2 was prepared according to reported method.[8] 


\section{PHYSICAL MEASUREMENTS}

Microanalysis of the Schiff base ligands and complexes were performed on a Perkin-Elmer(USA) 2400 Series II, elemental analyzer. The solutions of both ligands and complexes were prepared in HPLC grade $\mathrm{DMF}$ and electrical conductance measurements were performed using a Toshniwal Conductivity Bridge and a dip type cell calibrated with potassium chloride solutions. IR spectra for ligands and complexes were recorded in the range $400-4000 \mathrm{~cm}^{-1}$ on a Nicolet Magna IR 550 series II spectrophotometer using $\mathrm{KBr}$ pellets. ${ }^{1} \mathrm{H}$ NMR spectra were recorded in DMSO-d ${ }^{6}$ on a Bruker DRX-300 instrument, using TMS as an internal standard. Electronic spectra were recorded for solutions of ligands and complexes in DMF on a Shimadzu UV 3101 PC spectrophotometer. The thermogravimetric analysis is done on Mettler Toledo (Star Switzerland SDTA/TGA 851) Instrument, to determine the decomposition temperature of complexes. The $\mathrm{X}$ ray diffraction patterns have been recorded in $2 \theta$ range from 13 to $64^{\circ}$ on Philips (Holland) automated X-ray powder diffractometer. The operating target voltage was $35 \mathrm{kV}$, and the tube current was $20 \mathrm{~mA}$. The scanning speed was 0.5 20/min. Radiation used was $\mathrm{Cu}-\mathrm{k}$ wavelength $1.54056 \mathrm{~A}^{\circ}$ using monochromater for filtering $\beta$ - radiations and reducing noise due to white radiations and also to increase resolution. The values of interplaner spacing (d) corresponding

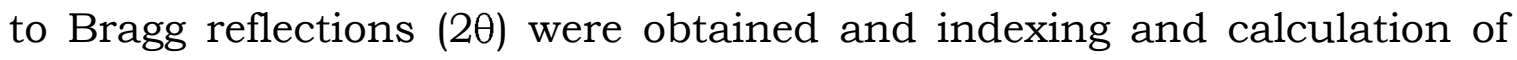
unit cell parameters were performed with the help of Powder-X Software.[9-11]

\section{SYNTHESIS OF SCHIFF BASE LIGANDS (1a-6a)}

An ethanolic solution of ketone/aldehyde such as salicyladehyde(sal); ohydroxybenzophenone (hbp) was mixed with an amine such as nicotinic acid hydrazide(NAH); benzoic acid hydrazide (BAH) and salicylic acid hydrazide (SAH) in 1: 1 molar ratio. The mixture was heated under reflux for 2-4 $\mathrm{h}$ and then cooled in an ice bath. The yellow crystals that 
separated out were filtered, washed several times with ethanol and then dried in vacuo. The ligands obtained are N'[(1Z)-1-(2hydroxyphenyl)phenylethylidene] nicotinoylhydrazine $\left(\mathrm{H}_{2} \mathrm{hbp}-\mathrm{NAH}\right.$ (1a), N'Salicylidene nicotinoylhydrazine $\left(\mathrm{H}_{2}\right.$ sal-NAH) (2a), N'[(1Z)-1-(2hydroxyphenyl) phenylethylidene] benzoylhydrazine ( $\left.\mathrm{H}_{2} \mathrm{hbp}-\mathrm{BAH}\right)$ (3a), N'Salicylidene benzoyllhydrazine ( $\mathrm{H}_{2}$ sal-BAH) (4a), N'[(1Z)-1-(2hydroxyphenylphenyl)ethylidene] salicyloylhydrazine ( $\left.\mathrm{H}_{2} \mathrm{hbp}-\mathrm{SAH}\right)$ (5a), N'Salicylidene salicyloylhydrazine $\left(\mathrm{H}_{2}\right.$ sal-SAH) (6a).

\section{SYNTHESIS OF MOLYBDENUM COMPLEXES (1b-6b)}

Bis(acetylacetonato)dioxomolybdenum(VI) [cis-( $\mathrm{MoO}_{2}$ (acac) $\left.)_{2}\right]$ undergoes ligand exchange with the Schiff bases in a suitable solvent and complexes of the type $\mathrm{MoO}_{2}(\mathrm{~L})(\mathrm{S})$ (where $\mathrm{LH}_{2}=$ Schiff base) are formed as follows : $\quad \Delta, 6 \mathrm{~h}$

$\mathrm{MoO}_{2}(\text { acac })_{2}+\mathrm{LH}_{2} \quad--------\rightarrow \quad$ cis- $-\mathrm{MoO}_{2}(\mathrm{~L})(\mathrm{S})+2$ acacH

$\mathrm{EtOH}$

Where, ' $\mathrm{S}$ ' is solvent

The cis- $\left[\mathrm{MoO}_{2}(\mathrm{~L})(\mathrm{solv})\right]$ complexes synthesized by reacting cis$\left[\mathrm{MoO}_{2}(\text { acac })_{2}\right]$ and the ligands (1a-6a) in ethanol are cis- $\left[\mathrm{MoO}_{2}\left(\mathrm{H}_{2} \mathrm{hbp}\right.\right.$ $\mathrm{NAH})(\mathrm{EtOH})] \quad(\mathbf{1 b})$, cis- $\left[\mathrm{MoO}_{2}(\mathrm{sal}-\mathrm{NAH})(\mathrm{EtOH})\right] \quad(\mathbf{2 b}), \quad$ cis- $\left[\mathrm{MoO}_{2}\left(\mathrm{H}_{2} \mathrm{hbp} \quad\right.\right.$ $\mathrm{BAH})(\mathrm{EtOH})] \quad(\mathbf{3 b}) \quad$ cis- $\left[\mathrm{MoO}_{2}(\mathrm{sal}-\mathrm{BAH})(\mathrm{EtOH})\right] \quad(\mathbf{4 b}), \quad$ cis- $\left[\mathrm{MoO}_{2}\left(\mathrm{H}_{2} \mathrm{hbp}-\right.\right.$ $\mathrm{SAH})(\mathrm{EtOH})](\mathbf{5 b})$ and cis- $\left[\mathrm{MoO}_{2}(\mathrm{sal}-\mathrm{SAH})\left(\mathrm{H}_{2} \mathrm{O}\right)\right](\mathbf{6 b})$.

\section{RESULTS AND DISCUSSION}

Bis(acetylacetonato) dioxomolybdenum(VI) [cis- $\mathrm{MoO}_{2}$ (acac) $)_{2}$, undergoes ligand exchange reaction with the Schiff bases (1a-6a) and complexes of the type $\left[\mathrm{MoO}_{2}(\mathrm{~L})(\mathrm{S})\right]$ (where $\mathrm{LH}_{2}=$ Schiff base) are formed as follows:

cis $-\mathrm{MoO}_{2}(\text { acac })_{2}+\mathrm{LH}_{2} \rightarrow$ cis $-\left[\mathrm{MoO}_{2}(\mathrm{~L})(\mathrm{S})\right]+2$ acacH

The analytical data presented in Table 1 show that the Schiff bases behave as dibasic, tridentate ligands, hence complete replacement of the 
bidentate acetylactone occurs under the reaction conditions and the solvent molecule occupies the sixth coordination position. The analytical data further support the formulation of the complexes as cis-[ $\left[\mathrm{MoO}_{2}\right.$ $(\mathrm{L})(\mathrm{S})]$. The structures for ligands (1a-6a )and for complexes (1b-6b )are shown in Figure 1 and 2. The molar conductance of the complexes in $\mathrm{DMF}$ is found to be in the ran ge 1.97-6.53 $\mathrm{ohm}^{-1} \mathrm{~cm}^{-1} \mathrm{~mol}^{-1}$ which indicates that they are all non-electrolytes. The ligands were tested separately for conductance and none of them indicated any measurable conductance.

\section{IR SPECTRA}

The IR spectra of the Schiff base ligands reveal the presence of $v \mathrm{NH}$ and $v \mathrm{C}=\mathrm{O}$ groups which are found to be absent in their corresponding $\mathrm{MoO}_{2}$ complexes and a new $\mathrm{C}-\mathrm{O}$ stetch was found, giving $>\mathrm{C}=\mathrm{N}-\mathrm{N}=\mathrm{C}<$ framework. This suggests occurrence of keto-imino tautomerisation of the ligand during complexation. The presence of two prominent bands in the complexes $(\mathbf{1 b}-\mathbf{6 b})$ in the regions $874-948 \mathrm{~cm}^{-1}$ and $848-913 \mathrm{~cm}^{-1}$ due to the $v_{\mathrm{sym}}(\mathrm{O}=\mathrm{Mo}=\mathrm{O})$ and $\operatorname{vasym}_{\mathrm{a}}(\mathrm{O}=\mathrm{Mo}=\mathrm{O})$ respectively, confirms the presence of cis- $\mathrm{MoO}_{2}{ }^{2+}$ centre.[12-15] It has been reported before that the $\mathrm{MoO}_{2}{ }^{2+}$ moiety prefers to form cis configuration due to the maximum utilization of the d-orbitals for the chemical bonding.[16-18] Further, the complexes (1b- 4b) exhibit an additional band in the region 3066-3453 $\mathrm{cm}^{-1}$ which is attributed to $v_{\mathrm{OH}}$ of $\mathrm{C}_{2} \mathrm{H}_{5} \mathrm{OH}$ molecule coordinated to the sixth site[19]. The complexes (5b and $\mathbf{6 b}$ ) exhibit a band in the range 1604-1624 $\mathrm{cm}^{-1}$ due to vor of coordinated $\mathrm{H}_{2} \mathrm{O}$ molecule to the sixth site.

Thus, the Schiff bases are found to be dibasic tridentate ligands having $-\mathrm{N}, \mathrm{O}, \mathrm{O}$ donor sites and their $\mathrm{MoO}_{2}$ complexes show octahedral geometry, having general formula $\left[\mathrm{MoO}_{2}(\mathrm{~L})(\mathrm{S})\right]$, the sixth site being occupied by the solvent molecule. 
The IR spectra of complexes (Table 2) also reveal that both symmetric and asymmetric stretching of cis $-\mathrm{MoO}_{2}$ in the complex formed with $\left(\mathrm{H}_{2} \mathrm{hbp}-\mathrm{NAH}\right)$ (1b) occur at a relatively higher energy (904 and 948cm-1) than the corresponding $\left(\mathrm{H}_{2}\right.$ sal-NAH) (2b), (913 and 920 $\mathrm{cm}^{-1}$ ). Similarly, both symmetric and asymmetric stretching of cis- $\mathrm{MoO}_{2}$ in the complex formed with $\left.\left(\mathrm{H}_{2} \mathrm{hbp}-\mathrm{BAH}\right)(\mathbf{3 b}) ;\left(\mathrm{H}_{2} \mathrm{hbp}-\mathrm{SAH}\right) \mathbf{( 5 b}\right)$ occur at a relatively higher energy (899 and $\left.923 \mathrm{~cm}^{-1}\right)$; (893 and $\left.943 \mathrm{~cm}^{-1}\right)$ respectively, than the corresponding ( $\mathrm{H}_{2}$ sal-BAH) (4b), (914 and $895 \mathrm{~cm}^{-}$ 1) and $\left(\mathrm{H}_{2} \mathrm{sal}-\mathrm{SAH}\right)(\mathbf{6 b})$, (891 and $\left.910 \mathrm{~cm}^{-1}\right)$ respectively. Moreover, the difference between symmetric and asymmetric frequencies of $\mathrm{O}=\mathrm{Mo}=\mathrm{O}$ is also less $\left(\Delta v=10-25 \mathrm{~cm}^{-1}\right)$ for complexes $(\mathbf{2 b}, \mathbf{4 b}, \mathbf{6 b})$ whereas this difference is more $\left(\Delta v=30-50 \mathbf{c m}^{-1}\right)$ for complex $(\mathbf{1 b} ; \mathbf{3 b} ; \mathbf{5 b})$. The differences in stretching frequencies of $\mathrm{O}=\mathrm{Mo}=\mathrm{O}$ of the cis $-\mathrm{MoO}_{2}$ moiety may be attributed to the progressively decreasing steric and electron withdrawing character of benzyl and $-\mathrm{H}$ on azomethine carbon in the complexes (1); $\mathbf{3 b} ; \mathbf{5 b})$ and $(\mathbf{2} \mathbf{b}, \mathbf{4 b}, \mathbf{6 b})$, respectively.

Further, appearance of $v(\mathrm{Mo}=\mathrm{O})$ symmetric and asymmetric peaks in complexes (1b and $\mathbf{2 b}$ ) occur at relatively high energy as compared to $(\mathbf{3 b}-\mathbf{6 b})$. This may be due to the presence of heterocyclic ring of nicotinic acid hydrazide (NAH) which is probably exerting distant effect on $\mathrm{O}=\mathrm{Mo}=\mathrm{O}$ bonds.

\section{ELECTRONIC SPECTRA}

In their electronic spectra, the ligands (1a-6a) showed peaks in the region 295-390 $\mathrm{nm}$ which can be attributed to intra ligand transitions. (Table-1) Similarly, the cis- $\mathrm{MoO}_{2}$ complexes displayed a broad band in the range 390-410nm which could be assigned to the ligand metal charge transfer transition (LMCT), between the lowest empty $\mathrm{d}^{0}$ orbital (dп) of molybdenum and the highest occupied ligand molecular orbital (p п) i.e. $\mathrm{Mo}(\mathrm{d} \pi) \leftarrow \mathrm{O}(\mathrm{p} \pi)$ transition. Several of the $\mathrm{MoO}_{2}$ complexes showed 
LMCT band in this range [20, 21]. As obivious for $4 \mathrm{~d}^{\circ}$ system, no $d-d$ transitions were expected in all the above complexes.

It has been observed that there is no significant effect on $\mathrm{Mo}(\mathrm{d} \pi) \leftarrow$ $\mathrm{O}(\mathrm{р} п)$ transition due to substitutional changes on hydrazide moiety (NAH, BAH and $\mathrm{SAH}$ ). On the other hand, the substituent present on azomethine carbon is found to exert a certain influence on the LMCT energy. In case of $-\mathrm{C}_{6} \mathrm{H}_{5}$ on azomethine carbon, an average increase of energy by 15-20 $\mathrm{nm}$ was observed in comparison to unsubstituted azomethine carbon $(\mathbf{2} \mathbf{b}, \mathbf{4 b}, \mathbf{6 b})$.

It appears that the energy of Mo $(\mathrm{d} \pi)$ level is progressively moved away from $\mathrm{O}(\mathrm{p} \pi)$ orbital as the electron donating character of the substituent $\left(-\mathrm{H},-\mathrm{C}_{6} \mathrm{H}_{5}\right)$ increases as shown below in the figure 3.

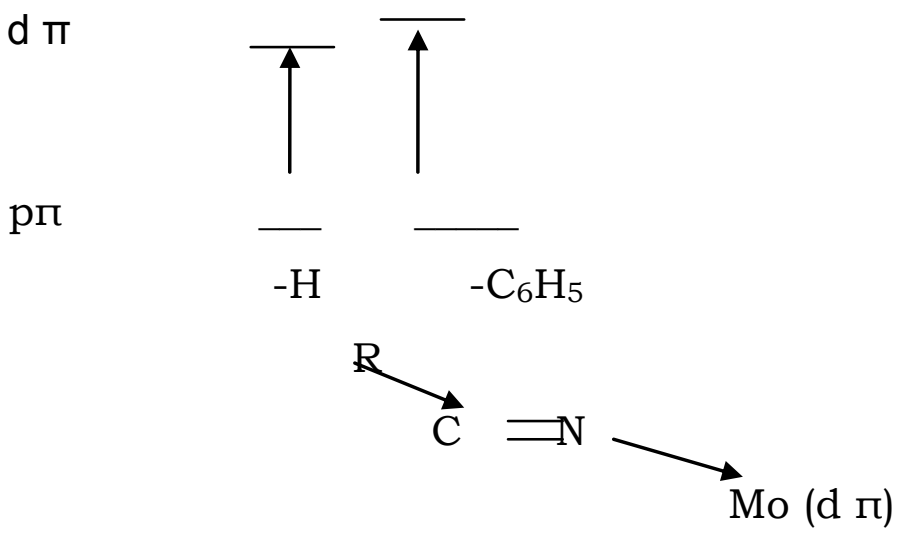

\section{Figure:3 Predicted Influence of substituent's on energy level of Mo(d r) orbital}

\section{${ }^{1}$ H NMR SPECTRA}

The IR data is further supported by the ${ }^{1} \mathrm{H}$ NMR spectral data (Table2). The ${ }^{1} \mathrm{H}$ NMR spectral data for the ligands (1a-6a) exhibit signals due to the phenolic $\mathrm{OH}$ protons and $\mathrm{NH}$ protons. The absence of these signals in the spectra of the complexes indicate coordination through the phenolic oxygen and enolization of the $-\mathrm{C}=\mathrm{O}$ of the aroyl keto group and consequent deprotonation. The ethanol coordination to molybdenum in 
complexes (1b- $\mathbf{4 b})$ is supported by the presence of the singlet, triplet and quartet of $\mathrm{OH}, \mathrm{CH}_{3}$ and $\mathrm{CH}_{2}$ at $11.1-12.93,1.06-1.42$ and 2.69-3.70 ppm respectively. The $\mathrm{H}_{2} \mathrm{O}$ coordination to molybdenum centre in complexes (5b - $\mathbf{6 b})$ is supported by the absence of the singlet, triplet and quartet of $\mathrm{OH}, \mathrm{CH}_{3}$ and $\mathrm{CH}_{2}$, respectively. Due to rapid exchange of protons in the coordinated $\mathrm{H}_{2} \mathrm{O}$, the signal corresponding to $\mathrm{H}_{2} \mathrm{O}$ could not be observed.

\section{THERMOGRAVIMETRIC ANALYSIS}

Thermal behavior of the complexes (1b-6b) was studied in the temperature range $30-800^{\circ} \mathrm{C}$, with increase of $10^{\circ} \mathrm{C} / \mathrm{min}$. The TG curves exhibit weight loss in four steps corresponding to the release of solvent molecule, two stage decomposition of Schiff base ligands and finally the formation of molybdenum trioxide (Table 3$)$. Complexes (1b, 2b, 3b and 4b) undergo a rapid weight loss from $80-102^{\circ} \mathrm{C}$ due to release of coordinated $\mathrm{C}_{2} \mathrm{H}_{5} \mathrm{OH}$ molecule and in the complexes (5b and $6 \mathrm{~b}$ ) the release of the coordinated $\mathrm{H}_{2} \mathrm{O}$ occurs in the temperature range 80$100^{\circ} \mathrm{C}$.

The Schiff base ligands decompose in two steps in the temperature range $210-770^{\circ} \mathrm{C}$, the first step corresponding to significant weight loss and the second showing a gradual weight loss. The \% weight loss value of the final residues was in accordance with that of molybdenum trioxide for all the complexes.

\section{X-RAY POWDER DIFFRACTION ANALYSIS}

The XRD data was obtained using Powder-X Software. The single crystals of complexes in DMF could not be obtained; hence, XRD patterns of the same are studied and reported. All the complexes were found to be crystalline and their X-ray powder diffractograms were 
collected. The lattice parameters and Miller's indices were computed. The indexing and calculation of unit cell parameters are performed with the help of Powder-X Software. The calculated and the observed $2 \theta$ value, the relative intensity, the inter-planar distance along with Miller's indices for corresponding angles are tabulated for the complexes.

On the basis of X-ray powder patterns and unit cell refinements, it is found that all the complexes adopt triclinic crystal system with $\mathrm{P}$ type lattice space group. The $\mathrm{hkl}$ indexing and space identification was carried out and complexes (1)-6b) adopt distorted octahedral geometry with triclinic space grouping The lattice constants were calculated, complex (1b) - $\mathbf{a}=7.9 \AA ; \mathrm{b}=10.1 \AA ; \mathrm{c}=10.35 \AA$ and $\alpha=80^{\circ}, \beta=79^{\circ} \gamma=$ $76^{\circ} ;(\mathbf{2 b})-a=7.99 \AA ; b=10.1 \AA ; c=10.42 \AA$ and $\alpha=81^{\circ} ; \beta=80^{\circ}$; $\gamma=79.2^{\circ} ;(4 \mathbf{b})-\mathrm{a}=8 \AA \mathrm{b}=10.2 \AA ; \mathrm{c}=10.35 \AA$ and $\alpha=80.9^{\circ}, \beta=$ $79.7^{\circ}, \gamma=78.1^{\circ}(6 \mathbf{b})-\mathrm{a}=7.88 \AA \mathrm{b}=10.45 \AA ; \mathrm{c}=10.1 \AA$ and $\alpha=$ $81.5^{\circ}, \beta=78.6^{\circ}, \gamma=78^{\circ}$. We have earlier reported the single crystal structure of $\operatorname{cis}-\mathrm{MoO}_{2}(\mathrm{~L})$ (Solv), where L = salicylaldehyde salicyloyl hydrazide) which was found to be triclinic with $\mathrm{P}$ type space group[22].

Thus, on the basis of elemental analysis, IR and TGA, all the complexes have their sixth coordinating site occupied by a solvent molecule either $\mathrm{C}_{2} \mathrm{H}_{5} \mathrm{OH}$ or $\mathrm{H}_{2} \mathrm{O}$. The Schiff base ligands coordinate through azomethine nitrogen, phenolic and enolic oxygen atoms $(-\mathrm{N}, \mathrm{O}$, O) confirming to distorted octahedral geometry.

\section{CONCLUSIONS}

It is evident from the above data, the Schiff base ligands behave as dibasic tridentate ligands and co-ordinate through phenolic oxygen, azomethine nitrogen and enolic oxygen atoms. The complexes are found to be monomers, non-electrolytes, diamagnetic and six co-ordinated. The complexes adopt triclinic crystal system with $\mathrm{P}$ type lattice space group. The sixth site in the complex is occupied by an ethanol/water group, which allows the binding and displacement of several substrate 
molecules during their use as a catalyst in the oxidation reactions. The use of the complexes of this type as catalyst for epoxidation of olefins is studied and reported elsewhere [23].

\section{REFERENCES}

Shailendra N.B, Gonzalez Garza M.T., Cruz-Vega D.E., Castro Garza J.C., Saleem K., Naqui F., Maurya M.R. and Azam A., (2002), Bioorg. \& Medicinal Chem. Lett., 12 , (6) , 869-871.

Rana A., Dinda R., Sengupta P., Ghosh S. and Falvello L.R.,Synthsis , (2002), Polyhedron, 21,1023-1030 .

Owaik T.G., Jerzykiewicz L., Sofczak J. aw.M and Kowski Zio J.J., (2003), Inorg. Chim. Acta, 356, 387-391.

Ambrazaik K., Pelech R., Milchert E., Dziemobowska T. and Rozwadowski Z., (2004), J. Mol. Catal. A- Chemical, 211, 9-16.

Valente A.A., Petrovski E., Branco L.C., Afonso C., Pillinger M., Lopes Romao A.D., Nunes C.D. and Goncalves I.S. ,(2004), J. Mol. Catal. A- Chemical, $218,5-11$.

Nunes C.D., Pillinger M., Valente A.A., Lopes A.D. and Goncalves I.S., (2003), Inorg. Chem. Comm., 6, 1228-1233.

Rao S. N., Mishra D. D., Maurya R.C., and Rao N. N., (1995), Bull. Chem. Soc. Jpn., 68, 1589-1592.

Chen G.J.J., McDonald J-W and Neuton W.E., (1976), Inorg.Chem., 15 ,pp.2612-2615.

Syed L.E. and Iskander M.F., (1971),J. Inorg. Nucl. Chem., 33 , 435-443.

W.H. Bragg and W.L. Bragg, (1933) "The Crystalline State”, Bell, London.

Bunn, (1945) “Chemical Crystallography”, Oxford University Press.

Azaroff and Buerger, (1958) ,"The powder Method”, McGraw Hill, London. 
Rao S.N., Munshi K.N., and Rao N.N., (1999), J.Mol.Catal. A- Chem., 145 , 203-210.

Rao S.N., Munshi K.N., Rao N.N., (2000), J Mol. Catal. A- Chem.,156, 205-211

Rao S.N., Kathale N., Munshi K.N., Rao N.N.,(2007), Inorg. Chim Acta., $360,4010-4016$.

N. Kathale, Rao S.N., Munshi K.N., Rao N.N., (2001), 31 , 859-871.

Rao S.N., Jaiswal M.N., Mishra D.D., Maurya R.C. and Rao N.N., (1993),Polyhedron, 12, 2045-2050.

Pickett C., Kumar S., Vella P.A. and Zubieta J., (1982).Inorg. Chem., 21, , 908-916.

Syamal A., Maurya M.R., (1986), Transition Met. Chem., 11, 255-258.

S.N. Rao, D. D. Mishra, R.C. Maurya, and N. N. Rao, (1995), Synth. React. Inorg. Met.-Org. Chem., 25, 437-449.

Maurya R. C., Mishra D. D., Rao S. N., Verma R. and Rao N. N.,( 1997) ,Indian J. Chem., 36A, , 599-601.

Rao S.N., Munshi K.N. ,Rao N.N., Badbhade M.M. and Suresh E., (1999), Polyhedron , 18 ,pp.2491-2497.

M. A. Katkar, S. N. Rao and H. D. Juneja, RSC Advances, 2, 8071-8078, 2012. 


\section{Table 1: Analytical Data and Electronic Spectral Data of Ligands (1a-6a) and Complexes (1b-6b)}

\begin{tabular}{|c|c|c|c|c|c|c|c|c|c|}
\hline \multirow[t]{2}{*}{$\begin{array}{l}\text { Sr. } \\
\text { no. }\end{array}$} & \multirow[t]{2}{*}{$\begin{array}{l}\text { Ligands/ } \\
\text { complexes }\end{array}$} & \multirow[t]{2}{*}{ Color } & \multirow[t]{2}{*}{ F.w. } & \multirow[t]{2}{*}{$\begin{array}{c}\begin{array}{c}\text { Yield } \\
(\%)\end{array} \\
\end{array}$} & \multirow[t]{2}{*}{$\begin{array}{l}\text { m.p } \\
\left({ }^{\circ} \mathrm{C}\right)\end{array}$} & \multicolumn{3}{|c|}{$\begin{array}{l}\text { Elemental Analysis(\%): } \\
\text { Calculated (found) }\end{array}$} & \multirow{2}{*}{$\begin{array}{c}\text { UV-Vis } \\
\boldsymbol{A} \mathbf{n m}\left(\underset{1}{\in} \mathbf{M}^{-1} \mathbf{c m}^{-}\right. \\
\text {1) }\end{array}$} \\
\hline & & & & & & $\mathbf{C}$ & $\mathbf{H}$ & $\mathbf{N}$ & \\
\hline $1 a$ & $\begin{array}{l}\mathrm{H}_{2} \mathrm{hbp}-\mathrm{NAH} \\
\mathrm{C}_{19} \mathrm{H}_{15} \mathrm{~N}_{3} \mathrm{O}_{2}\end{array}$ & Yellow & 317 & 87 & 190 & $\begin{array}{c}71.92 \\
(71.90)\end{array}$ & $\begin{array}{c}4.73 \\
(4.75) \\
\end{array}$ & $\begin{array}{c}13.24 \\
(13.25)\end{array}$ & $\begin{array}{l}295\left(1.7 \times 10^{4}\right) \\
330\left(1.7 \times 10^{4}\right)\end{array}$ \\
\hline 1b & $\begin{array}{l}\mathrm{MoO}_{2}(\mathrm{hbp}- \\
\mathrm{NAH}) \\
\mathrm{MoC}_{21} \mathrm{H}_{19} \mathrm{~N}_{3} \mathrm{O}_{5}\end{array}$ & een & 488.94 & 89 & 285 & $\begin{array}{l}51.54 \\
(51.24)\end{array}$ & $\begin{array}{c}3.88 \\
(4.02)\end{array}$ & $\begin{array}{c}8.89 \\
(8.79)\end{array}$ & $405\left(7.5 \times 10^{3}\right)$ \\
\hline $2 a$ & $\begin{array}{l}\mathrm{H}_{2} \text { sal-NAH; } \\
\mathrm{C}_{13} \mathrm{H}_{11} \mathrm{~N}_{3} \mathrm{O}_{2}\end{array}$ & Yellow & 241 & 85 & 160 & $\begin{array}{r}6 \\
6 \\
\end{array}$ & $\begin{array}{c}4.56 \\
(4.32)\end{array}$ & $\begin{array}{c}17.42 \\
(17.46)\end{array}$ & $\begin{array}{l}295\left(2.1 \times 10^{4}\right) \\
330\left(2.0 \times 10^{4}\right)\end{array}$ \\
\hline 2b & $\begin{array}{l}\mathrm{MoO}_{2} \text { (sal- } \\
\mathrm{NAH} \text {; } \\
\mathrm{MoC}_{15} \mathrm{H}_{15} \mathrm{~N}_{3} \mathrm{O}_{5}\end{array}$ & $n$ & 412.94 & 84 & 210 & $\begin{array}{c}43.58 \\
(43.97)\end{array}$ & $\begin{array}{c}3.63 \\
(3.65)\end{array}$ & $\begin{array}{l}10.17 \\
(10.2)\end{array}$ & $410\left(0.3 \times 10^{4}\right)$ \\
\hline $\mathbf{3 a}$ & $\begin{array}{l}\mathrm{H}_{2} \mathrm{hb} \\
\mathrm{C}_{20 \mathrm{H}}\end{array}$ & Cream & 6 & 7 & 220 & $(75$ & $\begin{array}{c}5.97 \\
(5.66)\end{array}$ & $\begin{array}{l}10.44 \\
(9.47)\end{array}$ & $\begin{array}{l}300\left(1.9 \times 10^{4}\right) \\
350\left(1.9 \times 10^{4}\right)\end{array}$ \\
\hline $\mathbf{3 b}$ & $\begin{array}{l}\mathrm{MoO}_{2}(\mathrm{hbp}- \\
\mathrm{BAH}) ; \\
\mathrm{MoC}_{22} \mathrm{H}_{20} \mathrm{~N}_{2} \mathrm{O}_{5}\end{array}$ & Green & 487.94 & 86 & 255 & $\begin{array}{l}45.08 \\
(45.82)\end{array}$ & $\begin{array}{c}4.09 \\
(4.12)\end{array}$ & $\begin{array}{l}5.73 \\
(5.8)\end{array}$ & $405\left(1.9 \times 10^{4}\right)$ \\
\hline $4 a$ & $\begin{array}{l}\mathrm{H}_{2} \text { sal-BAH; } \\
\mathrm{C}_{14} \mathrm{H}_{12} \mathrm{~N}_{2} \mathrm{O}_{2}\end{array}$ & Cream & 240 & 82.48 & 184 & $\begin{array}{c}70.0 \\
(69.82)\end{array}$ & $\begin{array}{c}5.00 \\
(4.78)\end{array}$ & $\begin{array}{l}11.66 \\
(10.79)\end{array}$ & $\begin{array}{l}280\left(1.6 \times 10^{4}\right) \\
330\left(2.9 \times 10^{4}\right)\end{array}$ \\
\hline 4b & $\begin{array}{l}\mathrm{MoO}_{2} \text { (sal- } \\
\mathrm{BAH}) ; \\
\mathrm{MoC}_{16} \mathrm{H}_{16} \mathrm{~N}_{2} \mathrm{O}_{5}\end{array}$ & Green & 4 & 89 & 289 & $\begin{array}{l}46.60 \\
(46.12)\end{array}$ & $\begin{array}{l}3.88 \\
(3.87)\end{array}$ & $\begin{array}{c}6.79 \\
(6.95)\end{array}$ & $410\left(0.3 \times 10^{4}\right)$ \\
\hline $5 \mathbf{a}$ & $\begin{array}{l}\mathrm{H}_{2} \mathrm{hbp}-\mathrm{SAH} \\
\mathrm{C}_{20} \mathrm{H}_{16} \mathrm{~N}_{2} \mathrm{O}_{3}\end{array}$ & $\mathrm{C}$ & 332 & 83.16 & 189 & $\begin{array}{c}71.28 \\
(71.66) \\
\end{array}$ & $\begin{array}{c}4.81 \\
(4.44) \\
\end{array}$ & $\begin{array}{c}8.43 \\
(8.55) \\
\end{array}$ & $\begin{array}{l}300\left(1.0 \times 10^{4}\right) \\
360\left(1.0 \times 10^{4}\right)\end{array}$ \\
\hline $5 \mathbf{b}$ & $\begin{array}{l}\mathrm{MoO}_{2}(\mathrm{hbp}- \\
\mathrm{SAH}) ; \\
\mathrm{MoC}_{20} \mathrm{H}_{16} \mathrm{~N}_{2} \mathrm{O}_{6}\end{array}$ & Green & 475.94 & 86 & 300 & $\begin{array}{c}50.42 \\
(50.35)\end{array}$ & $\begin{array}{r}3.36 \\
(3.51) \\
\end{array}$ & $\begin{array}{c}5.88 \\
(5.98)\end{array}$ & $405\left(1.0 \times 10^{4}\right)$ \\
\hline $6 a$ & $\begin{array}{l}\mathrm{H}_{2} \mathrm{sal}-\mathrm{SAH} ; \\
\mathrm{C}_{14} \mathrm{H}_{12} \mathrm{~N}_{2} \mathrm{O}_{3} \\
\end{array}$ & Yellow & 256 & 89 & 182 & $\begin{array}{c}65.6 \\
(65.83) \\
\end{array}$ & $\begin{array}{r}4.68 \\
(4.32) \\
\end{array}$ & $\begin{array}{c}5.46 \\
(5.11) \\
\end{array}$ & $\begin{array}{l}297\left(1.7 \times 10^{4}\right) \\
335\left(2.2 \times 10^{4}\right)\end{array}$ \\
\hline $6 b$ & $\begin{array}{l}\mathrm{MoO}_{2} \text { (sal- } \\
\mathrm{SAH}) \\
\mathrm{MoC}_{14} \mathrm{H}_{12} \mathrm{~N}_{2} \mathrm{O}_{6}\end{array}$ & Orange & 399.94 & 86 & 254 & $\begin{array}{l}42.00 \\
(41.90)\end{array}$ & $\begin{array}{c}3.00 \\
(2.65)\end{array}$ & $\begin{array}{c}7.00 \\
(6.95)\end{array}$ & $\begin{array}{c}410 \\
\left(1.95 \times 10^{4}\right)\end{array}$ \\
\hline
\end{tabular}


Table 2: IR and ${ }^{1} \mathrm{H}$ NMR Spectral Data of Ligands (1a-6a) and

Complexes (1b-6b)

\begin{tabular}{|c|c|c|c|c|c|c|c|c|}
\hline \multirow{2}{*}{$\begin{array}{l}\text { Sr } \\
\text { No }\end{array}$} & \multirow{2}{*}{$\begin{array}{l}\text { Ligands/ } \\
\text { Complexes }\end{array}$} & \multicolumn{6}{|c|}{ Infrared Data $\left(\mathrm{cm}^{-1}\right)$} & \multirow[t]{2}{*}{ 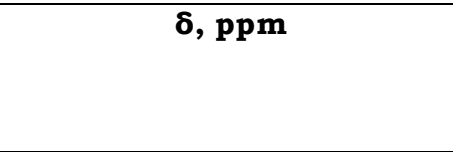 } \\
\hline & & $\begin{array}{l}\mathrm{v}(\mathbf{O} \\
\mathbf{H})\end{array}$ & $\begin{array}{c}v(\mathbf{N H} \\
\quad)\end{array}$ & $v(\mathbf{C}=\mathbf{N})$ & $v(\mathbf{C}=\mathbf{O}$ & $\begin{array}{l}v(\mathbf{C}- \\
\mathbf{O})\end{array}$ & $\begin{array}{c}v(\mathbf{M o}=\mathbf{O} \\
)\end{array}$ & \\
\hline $1 \mathbf{a}$ & $\begin{array}{c}\mathrm{H}_{2} \mathrm{hbp}- \\
\mathrm{NAH} ; \\
\mathrm{C}_{19} \mathrm{H}_{15} \mathrm{~N}_{3} \mathrm{O}_{2} \\
\end{array}$ & $\begin{array}{c}332 \\
2\end{array}$ & 3021 & 1660 & 1672 & $\begin{array}{ll}------ \\
\end{array}$ & $\begin{array}{ll}------ \\
\end{array}$ & $\begin{array}{l}11.70(\mathrm{~s}, \mathrm{OH}, 1 \mathrm{H}) ; \\
12.09(\mathrm{~s}, \mathrm{NH}, 1 \mathrm{H}) ; 6.97- \\
\text { 8.02m,ArH,13H). }\end{array}$ \\
\hline $\mathbf{1 b}$ & $\begin{array}{c}\mathrm{MoO}_{2}(\mathrm{hbp}- \\
\mathrm{NAH}) ; \\
\mathrm{MoC}_{21} \mathrm{H}_{19} \mathrm{~N} \\
{ }_{3} \mathrm{O}_{5}\end{array}$ & $\begin{array}{c}321 \\
1\end{array}$ & $\begin{array}{c}----- \\
-\end{array}$ & $\begin{array}{l}1625 \\
1594\end{array}$ & $\begin{array}{c}----- \\
-\end{array}$ & 1156 & $\begin{array}{l}948 \\
904\end{array}$ & $\begin{array}{l}11.70(\mathrm{~s}, \mathrm{OH}, 1 \mathrm{H}) ; 1.28-1.42(\mathrm{q},- \\
\left.\mathrm{CH}_{3}, 3 \mathrm{H}\right) ; 2.12- \\
2.19\left(\mathrm{t}, \mathrm{CH}_{2}, 2 \mathrm{H}\right) ; 6.97- \\
8.02 \mathrm{~m}, \mathrm{ArH}, 13 \mathrm{H}) .\end{array}$ \\
\hline $\mathbf{2 a}$ & $\begin{array}{l}\mathrm{H}_{2} \text { sal-NAH; } \\
\mathrm{C}_{13} \mathrm{H}_{11} \mathrm{~N}_{3} \mathrm{O}_{2}\end{array}$ & $\begin{array}{c}325 \\
0\end{array}$ & 3100 & 1580 & 1608 & $\begin{array}{ll}----- \\
\end{array}$ & $\begin{array}{ll}----- \\
\end{array}$ & $\begin{array}{l}\text { 11.43(s,OH, } 1 \mathrm{H}) \\
12.20(\mathrm{~s}, \mathrm{NH}, 1 \mathrm{H}) \\
8.3(\mathrm{~s}, \mathrm{CH}, 1 \mathrm{H}) ; 6.87- \\
8.75(\mathrm{~m}, \mathrm{ArH}, 8 \mathrm{H})\end{array}$ \\
\hline $2 \mathbf{b}$ & $\begin{array}{c}\mathrm{MoO}_{2}(\mathrm{sal}- \\
\mathrm{NAH}) \\
\mathrm{MoC}_{15} \mathrm{H}_{15} \mathrm{~N} \\
{ }_{3} \mathrm{O}_{5} \\
\end{array}$ & $\begin{array}{c}331 \\
5\end{array}$ & $\begin{array}{c}---- \\
-\end{array}$ & $\begin{array}{l}1560 \\
1535\end{array}$ & $\begin{array}{c}---- \\
-\end{array}$ & 1151 & $\begin{array}{l}920 \\
913\end{array}$ & $\begin{array}{l}12.91(\mathrm{~s}, \mathrm{OH}, 1 \mathrm{H}) ; \\
8.34(\mathrm{~s}, \mathrm{CH}, 1 \mathrm{H}) 1.06-1.17(\mathrm{q},- \\
\left.\mathrm{CH}_{3}, 3 \mathrm{H}\right) ; 3.16-3.31\left(\mathrm{t}, \mathrm{CH}_{2}\right. \\
, 2 \mathrm{H}) ; 6.87-8.75(\mathrm{~m}, \mathrm{ArH}, 8 \mathrm{H}) .\end{array}$ \\
\hline $3 a$ & $\begin{array}{c}\mathrm{H}_{2} \mathrm{hbp}- \\
\mathrm{BAH} ; \\
\mathrm{C}_{20} \mathrm{H}_{16} \mathrm{~N}_{2} \mathrm{O}_{2} \\
\end{array}$ & $\begin{array}{c}354 \\
0\end{array}$ & 3063 & 1608 & 1652 & $\begin{array}{l}----- \\
--\end{array}$ & ------- & $\begin{array}{l}10.92(\mathrm{~s}, \mathrm{OH}, 1 \mathrm{H}) ; 13.1(\mathrm{~s}, \mathrm{NH}, 1 \\
\mathrm{H}) ; 7.08-8.03(\mathrm{~m}, \mathrm{ArH}, 14 \mathrm{H}) .\end{array}$ \\
\hline $3 \mathbf{b}$ & $\begin{array}{l}\mathrm{MoO}_{2}(\mathrm{hbp}- \\
\mathrm{BAH} ; \\
\mathrm{MoC}_{22} \mathrm{H}_{20} \mathrm{~N} \\
{ }_{2} \mathrm{O}_{5}\end{array}$ & $\begin{array}{c}306 \\
6\end{array}$ & $\begin{array}{c}----- \\
-\end{array}$ & $\begin{array}{l}1595 \\
1553\end{array}$ & $\begin{array}{l}----- \\
--\end{array}$ & 1177 & $\begin{array}{l}923 \\
899\end{array}$ & $\begin{array}{l}11.1(\mathrm{~s}, \mathrm{OH}, 1 \mathrm{H}) ; 1.21-1.29(\mathrm{q},- \\
\left.\mathrm{CH}_{3}, 3 \mathrm{H}\right) ; 2.56-2.57(\mathrm{t},- \\
\left.\mathrm{CH}_{2}, 3 \mathrm{H}\right) ; 7.01- \\
8.05(\mathrm{~m}, \mathrm{ArH}, 14 \mathrm{H})\end{array}$ \\
\hline $4 a$ & $\begin{array}{l}\mathrm{H}_{2} \text { sal-BAH; } \\
\mathrm{C}_{14} \mathrm{H}_{12} \mathrm{~N}_{2} \mathrm{O}_{2}\end{array}$ & $\begin{array}{c}363 \\
1\end{array}$ & 3260 & 1622 & 1672 & $\begin{array}{c}------ \\
-\end{array}$ & ------- & $\begin{array}{l}11.58(\mathrm{~s}, \mathrm{OH}, 1 \mathrm{H}) ; 11.82(\mathrm{~s}, \mathrm{NH}, 1 \\
\mathrm{H}) ; 8.53(\mathrm{~s}, \mathrm{CH}, 1 \mathrm{H}) ; 6.86- \\
\text { 8.5(m,ArH,9H). }\end{array}$ \\
\hline $4 b$ & $\begin{array}{c}\mathrm{MoO}_{2}(\mathrm{sal}- \\
\mathrm{BAH} ; \\
\mathrm{MoC}_{16} \mathrm{H}_{16} \mathrm{~N} \\
{ }_{2} \mathrm{O}_{5}\end{array}$ & $\begin{array}{c}320 \\
0\end{array}$ & $\begin{array}{c}----- \\
--\end{array}$ & $\begin{array}{l}1612 \\
1556\end{array}$ & $\begin{array}{l}----- \\
--\end{array}$ & 1153 & $\begin{array}{l}914 \\
895\end{array}$ & $\begin{array}{l}11.58(\mathrm{~s}, \mathrm{OH}, 1 \mathrm{H}) ; \\
8.53(\mathrm{~s}, \mathrm{CH}, 1 \mathrm{H}) ; 1.37-1.41(\mathrm{q},- \\
\left.\mathrm{CH}_{3}, 3 \mathrm{H}\right) ; 3.38-3.44(\mathrm{t},- \\
\left.\mathrm{CH}_{2}, 3 \mathrm{H}\right) ; 6.94- \\
8.12(\mathrm{~m}, \mathrm{ArH}, 9 \mathrm{H}) .\end{array}$ \\
\hline $\mathbf{5 a}$ & $\begin{array}{c}\mathrm{H}_{2} \mathrm{hbp}-\mathrm{SAH} \\
; \\
\mathrm{C}_{20} \mathrm{H}_{16} \mathrm{~N}_{2} \mathrm{O}_{3}\end{array}$ & $\begin{array}{c}334 \\
3 \\
332 \\
0 \\
\end{array}$ & 3060 & 1600 & 1648 & ----- & ------ & $\begin{array}{l}11.52(\mathrm{~s}, \mathrm{OH}, 2 \mathrm{H}) \\
10.4(\mathrm{~s}, \mathrm{NH}, 1 \mathrm{H}) ; 7.54-8.28(\mathrm{~m} \\
\text {,ArH,13H). }\end{array}$ \\
\hline $5 \mathbf{b}$ & $\begin{array}{l}\mathrm{MoO}_{2}(\mathrm{hbp}- \\
\mathrm{SAH}^{-} \\
\mathrm{MoC}_{20} \mathrm{H}_{16} \mathrm{~N} \\
{ }_{2} \mathrm{O}_{6}\end{array}$ & $\begin{array}{c}334 \\
3 \\
162 \\
4 \\
\end{array}$ & $\begin{array}{ll}---- \\
\end{array}$ & $\begin{array}{c}1595 . \\
01 \\
1604 \\
45\end{array}$ & $\begin{array}{ll}---- \\
\end{array}$ & 1248 & $\begin{array}{c}943 ; 8 \\
93\end{array}$ & $\begin{array}{l}11.5(\mathrm{~s}, \mathrm{OH}, 1 \mathrm{H}) ; 1.4- \\
1.64\left(\mathrm{q}, \mathrm{CH}_{3}, 3 \mathrm{H}\right) ; 2,81- \\
2.98\left(\mathrm{t}, \mathrm{CH}_{2}, 2 \mathrm{H}\right) ; 7.4-7.81(\mathrm{~m} \\
\text {,ArH, } 13 \mathrm{H}) .\end{array}$ \\
\hline $6 \mathbf{a}$ & $\begin{array}{l}\mathrm{H}_{2} \text { sal-SAH; } \\
\mathrm{C}_{14} \mathrm{H}_{12} \mathrm{~N}_{2} \mathrm{O}_{3}\end{array}$ & $\begin{array}{c}329 \\
0 \\
320 \\
0\end{array}$ & 3050 & 1618 & 1655 & ----- & ----- & $\begin{array}{l}11.97(\mathrm{~s}, \mathrm{OH}, 2 \mathrm{H}) ; \\
11.40(\mathrm{~s}, \mathrm{NH}, 1 \mathrm{H}) ; 8.6 \\
(\mathrm{~s}, \mathrm{CH}, 1 \mathrm{H}) 6.8-7.93(\mathrm{~m}, \mathrm{ArH} \\
, 8 \mathrm{H})\end{array}$ \\
\hline $6 b$ & $\begin{array}{l}\mathrm{MoO}_{2}(\mathrm{sal}- \\
\mathrm{SAH}) ; \\
\mathrm{MoC}_{14} \mathrm{H}_{12} \mathrm{~N} \\
{ }_{2} \mathrm{O}_{6}\end{array}$ & $\begin{array}{c}329 \\
0 \\
162 \\
4\end{array}$ & $\begin{array}{c}----- \\
-\end{array}$ & $\begin{array}{l}1601 \\
1552\end{array}$ & $\begin{array}{l}----- \\
--\end{array}$ & 1246 & $\begin{array}{l}891 \\
910\end{array}$ & $\begin{array}{l}11.31(\mathrm{~s}, \mathrm{OH}, 1 \mathrm{H}) ; \\
8.68(\mathrm{~s}, \mathrm{CH}, 1 \mathrm{H}) ; 6.90-8.68( \\
\mathrm{m}, \mathrm{ArH}, 8 \mathrm{H})\end{array}$ \\
\hline
\end{tabular}


Table 3: Thermal Decomposition Data of complexes (1b-6b)

\begin{tabular}{|c|c|c|c|c|c|c|c|c|c|c|c|c|c|}
\hline \multirow{3}{*}{$\begin{array}{l}\text { Sr } \\
\dot{\text { n }} \\
\text { o. }\end{array}$} & \multirow{3}{*}{$\begin{array}{c}\mathbf{T}_{\mathrm{d}} \\
\left({ }^{\circ} \mathbf{C}\right)\end{array}$} & \multicolumn{12}{|c|}{ \% Weight Loss } \\
\hline & & \multicolumn{2}{|c|}{$\begin{array}{c}1 \mathrm{~b} \\
\mathrm{MoO}_{2}(\mathrm{hbp}- \\
\mathrm{NAH} \\
\mathrm{MoC}_{21} \mathrm{H}_{19} \mathrm{~N} \\
{ }_{3} \mathrm{O}_{5} \\
\end{array}$} & \multicolumn{2}{|c|}{$\begin{array}{c}2 b \\
\mathrm{MoO}_{2} \text { (Sal- } \\
\mathrm{NAH} \\
\mathrm{MoC}_{15} \mathrm{H}_{15} \\
\mathrm{~N}_{3} \mathrm{O}_{5} \\
\end{array}$} & \multicolumn{2}{|c|}{$\begin{array}{c}3 b \\
\mathrm{MoO}_{2}(\mathrm{hbp}- \\
\mathrm{BAH}) \\
\mathrm{MoC}_{22} \mathrm{H}_{20} \\
\mathrm{~N}_{2} \mathrm{O}_{5} \\
\end{array}$} & \multicolumn{2}{|c|}{$\begin{array}{c}4 b \\
\mathrm{MoO}_{2} \text { (sal- } \\
\mathrm{BAH} \\
\mathrm{MoC}_{16} \mathrm{H}_{16} \\
\mathrm{~N}_{2} \mathrm{O}_{5} \\
\end{array}$} & \multicolumn{2}{|c|}{$\begin{array}{c}5 \mathbf{b} \\
\mathrm{MoO}_{2}(\mathbf{h b p}- \\
\mathrm{SAH}) \\
\mathrm{MoC}_{20} \mathrm{H}_{16} \\
\mathrm{~N}_{2} \mathrm{O}_{6} \\
\end{array}$} & \multicolumn{2}{|c|}{$\begin{array}{c}6 b \\
\mathrm{MoO}_{2}(\mathrm{sal}- \\
\mathrm{SAH}) \\
\mathrm{MoC}_{14} \mathrm{H}_{12} \\
\mathrm{~N}_{2} \mathrm{O}_{6} \\
\end{array}$} \\
\hline & & $\begin{array}{c}\text { Cal } \\
\text { c. }\end{array}$ & Obs & $\begin{array}{c}\text { Cal } \\
\text { c. }\end{array}$ & Obs & $\begin{array}{c}\text { Cal } \\
\text { c. }\end{array}$ & Obs & $\begin{array}{c}\text { Cal } \\
\text { c. }\end{array}$ & Obs & $\begin{array}{c}\text { Cal } \\
\text { c. }\end{array}$ & Obs & $\begin{array}{c}\text { Cal } \\
\text { c. }\end{array}$ & Obs \\
\hline 1 & $\begin{array}{c}80- \\
102 \\
{ }^{\circ} \mathrm{C}\end{array}$ & $\begin{array}{c}9.4 \\
0\end{array}$ & $\begin{array}{c}9.2 \\
5\end{array}$ & 9.40 & 9.25 & 9.42 & 8.73 & $\begin{array}{c}11.1 \\
6\end{array}$ & $\begin{array}{c}10.8 \\
8\end{array}$ & 3.70 & 3.52 & $\begin{array}{c}4.5 \\
0\end{array}$ & 4.20 \\
\hline 2 & $\begin{array}{c}296 \\
- \\
420 \\
{ }^{\circ} \mathrm{C} \\
\end{array}$ & $\begin{array}{l}44 . \\
02\end{array}$ & $\begin{array}{l}43 . \\
82\end{array}$ & $\begin{array}{c}44.0 \\
2\end{array}$ & $\begin{array}{c}43.8 \\
2\end{array}$ & $\begin{array}{c}44.1 \\
2\end{array}$ & $\begin{array}{c}44.4 \\
5\end{array}$ & $\begin{array}{c}32.5 \\
0\end{array}$ & $\begin{array}{c}33.2 \\
1\end{array}$ & $\begin{array}{c}42.5 \\
8\end{array}$ & 42 & $\begin{array}{r}31 . \\
15\end{array}$ & $\begin{array}{c}32.1 \\
5\end{array}$ \\
\hline 3 & $\begin{array}{c}420 \\
- \\
770 \\
{ }^{\circ} \mathrm{C}\end{array}$ & $\begin{array}{l}48 . \\
39\end{array}$ & $\begin{array}{l}47 . \\
71\end{array}$ & $\begin{array}{c}48.3 \\
9\end{array}$ & $\begin{array}{c}47.6 \\
2\end{array}$ & $\begin{array}{c}48.1 \\
8\end{array}$ & $\begin{array}{c}48.7 \\
2\end{array}$ & $\begin{array}{c}48.1 \\
8\end{array}$ & $\begin{array}{c}47.4 \\
7\end{array}$ & $\begin{array}{c}51.3 \\
4\end{array}$ & $\begin{array}{c}50.5 \\
0\end{array}$ & $\begin{array}{l}51 . \\
34\end{array}$ & $\begin{array}{c}52.1 \\
0\end{array}$ \\
\hline
\end{tabular}

\section{$T_{d}$ - Decomposition temperature}

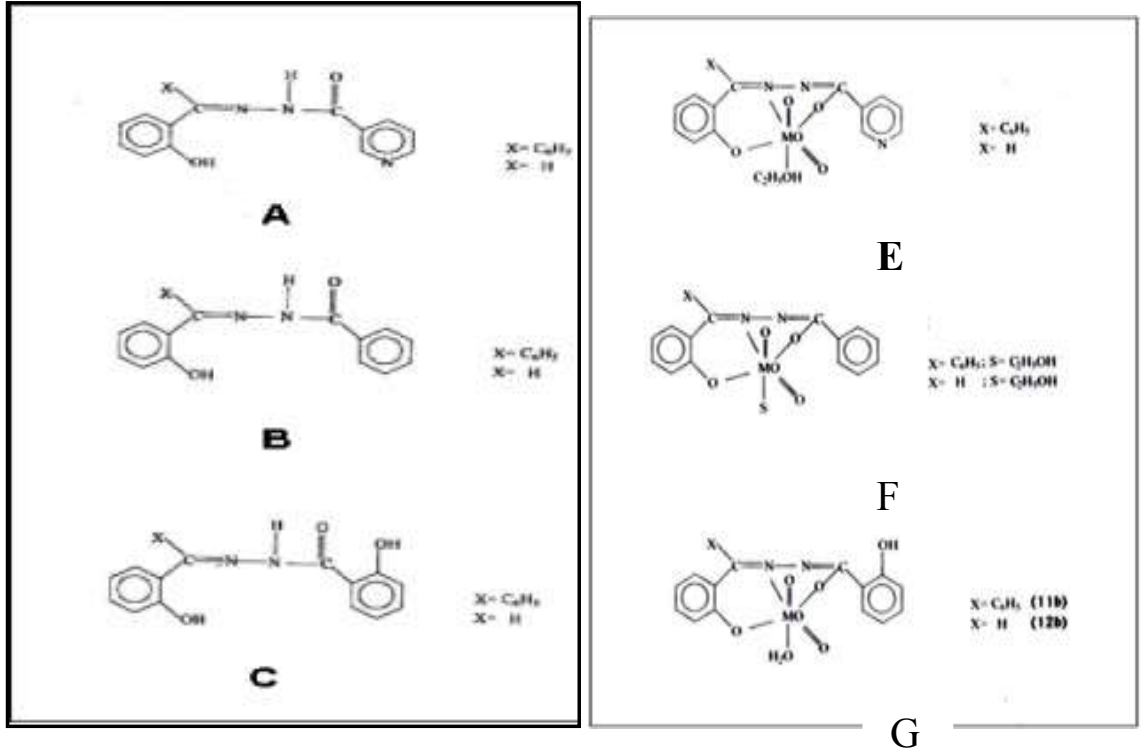

Fig1: A- Proposed structure for $1 \mathrm{a}$ and $2 \mathrm{a}$

Fig 2: E- Proposed structure for $1 b$ and $2 b$

B- Proposed structure for $3 a$ and $4 a$

F- Proposed structure for

$3 b$ and $4 b$

$$
\text { C- Proposed structure for } 5 a \text { and } 6 a
$$

G- Proposed structure for

$5 b$ and $6 b$ 
Table 1 : Analytical Data of Ligands (1a-6a) and Complexes (1b-6b)

\begin{tabular}{|c|c|c|c|c|c|c|c|c|}
\hline $\begin{array}{l}\text { Sr } \\
\text {. }\end{array}$ & $\begin{array}{l}\text { Ligands/ } \\
\text { complexes }\end{array}$ & Color & F.W. & $\begin{array}{c}\text { Yiel } \\
\text { d }\end{array}$ & $\mathbf{m}$ & \multicolumn{3}{|c|}{$\begin{array}{l}\text { Elemental Analysis(\%): } \\
\text { Calculated (found) }\end{array}$} \\
\hline & & & & & & C & $\mathbf{H}$ & $\mathbf{N}$ \\
\hline $1 \mathbf{a}$ & $\begin{array}{l}\mathrm{H}_{2} \mathrm{hbp}- \\
\mathrm{NAH} ; \mathrm{C}_{19} \mathrm{H}_{15} \mathrm{~N}_{3} \mathrm{O}_{2}\end{array}$ & $\begin{array}{l}\text { Yello } \\
\text { w }\end{array}$ & 317 & 87 & $\begin{array}{c}19 \\
0\end{array}$ & $\begin{array}{c}71.92 ;(71 . \\
90)\end{array}$ & $\begin{array}{c}4.73 ;(4.7 \\
5)\end{array}$ & $\begin{array}{c}13.24 ;(13 . \\
25)\end{array}$ \\
\hline 1b & $\begin{array}{l}\mathrm{MoO}_{2}\left(\mathrm{hbp}^{-}\right. \\
\mathrm{NAH} ; \mathrm{MoC}_{21} \mathrm{H}_{19} \\
\mathrm{~N}_{3} \mathrm{O}_{5}\end{array}$ & Green & $\begin{array}{c}488.9 \\
4\end{array}$ & 89 & $\begin{array}{c}28 \\
5\end{array}$ & $\begin{array}{l}51.54 ;(51 . \\
24)\end{array}$ & $\begin{array}{l}3.88 ;(4.0 \\
2)\end{array}$ & $8.89 ;(8.79)$ \\
\hline $2 \mathbf{a}$ & $\begin{array}{l}\mathrm{H}_{2} \text { sal- } \\
\mathrm{NAH} ; \mathrm{C}_{13} \mathrm{H}_{11} \mathrm{~N}_{3} \mathrm{O}_{2}\end{array}$ & $\begin{array}{l}\text { Yello } \\
\text { w }\end{array}$ & 241 & 85 & $\begin{array}{c}16 \\
0\end{array}$ & $\begin{array}{c}64.73 ;(65 . \\
83)\end{array}$ & $\begin{array}{l}4.56 ;(4.3 \\
2)\end{array}$ & $\begin{array}{c}17.42 ;(17 . \\
46)\end{array}$ \\
\hline $2 \mathbf{b}$ & $\begin{array}{l}\mathrm{MoO}_{2}\left(\mathrm{sal}_{-}\right. \\
\mathrm{NAH} ; \mathrm{MoC}_{15} \mathrm{H}_{15} \\
\mathrm{~N}_{3} \mathrm{O}_{5}\end{array}$ & Green & $\begin{array}{c}412.9 \\
4\end{array}$ & 84 & $\begin{array}{c}21 \\
0\end{array}$ & $\begin{array}{l}43.58 ;(43 . \\
97)\end{array}$ & $\begin{array}{c}3.63 ;(3.6 \\
5)\end{array}$ & $\begin{array}{l}10.17 ;(10 . \\
2)\end{array}$ \\
\hline $\mathbf{3 a}$ & $\begin{array}{l}\mathrm{H}_{2} \mathrm{hbp}-\mathrm{BAH} \\
\mathrm{C}_{20} \mathrm{H}_{16} \mathrm{~N}_{2} \mathrm{O}_{2}\end{array}$ & $\begin{array}{l}\text { Crea } \\
\mathrm{m}\end{array}$ & 316 & $\begin{array}{c}82.8 \\
7\end{array}$ & $\begin{array}{c}22 \\
0\end{array}$ & $\begin{array}{l}75.94(75.7 \\
6)\end{array}$ & $\begin{array}{c}5.97(5.6 \\
6)\end{array}$ & 10.44(9.47 \\
\hline $\mathbf{3 b}$ & $\begin{array}{l}\mathrm{MoO}_{2}(\mathrm{hbp}- \\
\mathrm{BAH} ; \mathrm{MoC}_{22} \mathrm{H}_{20} \\
\mathrm{~N}_{2} \mathrm{O}_{5}\end{array}$ & Green & $\begin{array}{c}487.9 \\
4\end{array}$ & 86 & $\begin{array}{c}25 \\
5\end{array}$ & $\begin{array}{l}45.08(45.8 \\
2)\end{array}$ & $\begin{array}{l}4.09(4.1 \\
2)\end{array}$ & $5.73(5.8)$ \\
\hline $4 a$ & $\begin{array}{l}\mathrm{H}_{2} \text { sal- } \\
\mathrm{BAH} ; \mathrm{C}_{14} \mathrm{H}_{12} \mathrm{~N}_{2} \mathrm{O}_{2}\end{array}$ & $\begin{array}{c}\text { Crea } \\
\mathrm{m}\end{array}$ & 240 & $\begin{array}{c}82.4 \\
8\end{array}$ & $\begin{array}{c}18 \\
4\end{array}$ & $\begin{array}{c}70.0(69.82 \\
)\end{array}$ & $\begin{array}{c}5.00(4.7 \\
8)\end{array}$ & $\begin{array}{l}11.66(10.7 \\
9)\end{array}$ \\
\hline 4b & $\begin{array}{l}\mathrm{MoO}_{2}\left(\mathrm{sal}^{-}\right. \\
\mathrm{BAH}) ; \mathrm{MoC}_{16} \mathrm{H}_{16} \\
\mathrm{~N}_{2} \mathrm{O}_{5}\end{array}$ & Green & $\begin{array}{c}411.9 \\
4\end{array}$ & 89 & $\begin{array}{c}28 \\
9\end{array}$ & $\begin{array}{c}46.60(46.1 \\
2)\end{array}$ & $\begin{array}{c}3.88(3.8 \\
7)\end{array}$ & $6.79(6.95)$ \\
\hline $5 \mathbf{5 a}$ & $\begin{array}{l}\mathrm{H}_{2} \mathrm{hbp}- \\
\mathrm{SAH} ; \mathrm{C}_{20} \mathrm{H}_{16} \mathrm{~N}_{2} \mathrm{O}_{3}\end{array}$ & $\begin{array}{c}\text { Crea } \\
\mathrm{m}\end{array}$ & 332 & $\begin{array}{c}83.1 \\
6\end{array}$ & $\begin{array}{c}18 \\
9 \\
\end{array}$ & $\begin{array}{c}71.28(71.6 \\
6)\end{array}$ & $\begin{array}{c}4.81(4.4 \\
4)\end{array}$ & $8.43(8.55)$ \\
\hline $5 \mathbf{b}$ & $\begin{array}{l}\mathrm{MoO}_{2}(\mathrm{hbp}- \\
\mathrm{SAH}) ; \mathrm{MoC}_{20} \mathrm{H}_{16} \mathrm{~N} \\
{ }_{2} \mathrm{O}_{6}\end{array}$ & Green & $\begin{array}{c}475.9 \\
4\end{array}$ & 86 & $\begin{array}{c}30 \\
0\end{array}$ & $\begin{array}{c}50.42(50.3 \\
5)\end{array}$ & $\begin{array}{c}3.36(3.5 \\
1)\end{array}$ & $5.88(5.98)$ \\
\hline $6 a$ & $\begin{array}{l}\mathrm{H}_{2} \text { sal- } \\
\mathrm{SAH} ; \mathrm{C}_{14} \mathrm{H}_{12} \mathrm{~N}_{2} \mathrm{O}_{3}\end{array}$ & $\begin{array}{l}\text { Yello } \\
\text { w }\end{array}$ & 256 & 89 & $\begin{array}{c}18 \\
2\end{array}$ & $\begin{array}{c}65.6(65.83 \\
)\end{array}$ & $\begin{array}{c}4.68(4.3 \\
2)\end{array}$ & $5.46(5.11)$ \\
\hline $6 b$ & $\begin{array}{l}\mathrm{MoO}_{2} \text { (sal-SAH); } \\
\mathrm{MoC}_{14} \mathrm{H}_{12} \mathrm{~N}_{2} \mathrm{O}_{6}\end{array}$ & $\begin{array}{c}\text { Oran } \\
\text { ge }\end{array}$ & $\begin{array}{c}399.9 \\
4 \\
\end{array}$ & 86 & $\begin{array}{c}25 \\
4 \\
\end{array}$ & $\begin{array}{c}42.00(41.9 \\
0)\end{array}$ & $\begin{array}{c}3.00(2.6 \\
5) \\
\end{array}$ & $7.00(6.95)$ \\
\hline
\end{tabular}


Table 2: IR Spectral Data of Ligands (1a-6a) and Complexes (1b-6b)

\begin{tabular}{|c|c|c|c|c|c|c|c|}
\hline \multirow{2}{*}{$\begin{array}{l}\text { Sr. } \\
\text { No. }\end{array}$} & \multirow[t]{2}{*}{ Ligands/ Complexes } & \multicolumn{6}{|c|}{ Infrared Data $\left(\mathrm{cm}^{-1}\right)$} \\
\hline & & $v(\mathbf{O H})$ & $v(\mathbf{N H})$ & $v(\mathbf{C}=\mathbf{N})$ & $v(\mathbf{C}=0)$ & $v(\mathbf{C}-\mathbf{O})$ & $v(\mathbf{M o}=0)$ \\
\hline 1a & $\begin{array}{c}\mathrm{H}_{2} \mathrm{hbp}_{-} \\
\mathrm{NAH} ; \mathrm{C}_{19} \mathrm{H}_{15} \mathrm{~N}_{3} \mathrm{O}_{2}\end{array}$ & 3322 & 3021 & 1660 & 1672 & ----- & $\begin{array}{l}----- \\
\end{array}$ \\
\hline 1b & $\begin{array}{c}\mathrm{MoO}_{2}\left(\mathrm{hbp}^{-}\right. \\
\mathrm{NAH}) ; \mathrm{MoC}_{21} \mathrm{H}_{19} \mathrm{~N}_{3} \mathrm{O}_{5}\end{array}$ & 3211 & ------- & $1625 ; 1594$ & ------- & 1156 & $948 ; 904$ \\
\hline $\mathbf{2 a}$ & $\mathrm{H}_{2}$ sal-NAH; $\mathrm{C}_{13} \mathrm{H}_{11} \mathrm{~N}_{3} \mathrm{O}_{2}$ & 3250 & 3100 & 1580 & 1608 & $\begin{array}{c}------ \\
\end{array}$ & ------ \\
\hline $\mathbf{2 b}$ & $\begin{array}{c}\mathrm{MoO}_{2}\left(\mathrm{sal}_{-}\right. \\
\mathrm{NAH}) ; \mathrm{MoC}_{15} \mathrm{H}_{15} \mathrm{~N}_{3} \mathrm{O}_{5}\end{array}$ & 3315 & ------- & $1560 ; 1535$ & ------- & 1151 & $920 ; 913$ \\
\hline $\mathbf{3 a}$ & $\begin{array}{l}\mathrm{H}_{2} \mathrm{hbp}-\mathrm{BAH} \\
; \mathrm{C}_{20} \mathrm{H}_{16} \mathrm{~N}_{2} \mathrm{O}_{2}\end{array}$ & 3540 & 3063 & 1608 & 1652 & $\overline{-----}$ & ------- \\
\hline $\mathbf{3 b}$ & $\begin{array}{c}\mathrm{MoO}_{2}\left(\mathrm{hbp}^{-}\right. \\
\mathrm{BAH}) ; \mathrm{MoC}_{22} \mathrm{H}_{20} \mathrm{~N}_{2} \mathrm{O}_{5}\end{array}$ & 3066 & ------ & $1595 ; 1553$ & -------- & 1177 & $923 ; 899$ \\
\hline $4 a$ & $\mathrm{H}_{2}$ sal-BAH; $\mathrm{C}_{14} \mathrm{H}_{12} \mathrm{~N}_{2} \mathrm{O}_{2}$ & 3631 & 3260 & 1622 & 1672 & $\begin{array}{ll}------- \\
\end{array}$ & ------- \\
\hline 4b & $\begin{array}{l}\mathrm{MoO}_{2}(\mathrm{sal}-\mathrm{BAH}) \\
; \mathrm{MoC}_{16} \mathrm{H}_{16} \mathrm{~N}_{2} \mathrm{O}_{5}\end{array}$ & 3200 & ------- & $1612 ; 1556$ & ------- & 1153 & $914 ; 895$ \\
\hline $\mathbf{5 a}$ & $\begin{array}{l}\mathrm{H}_{2} \mathrm{hbp}-\mathrm{SAH} \\
; \mathrm{C}_{20} \mathrm{H}_{16} \mathrm{~N}_{2} \mathrm{O}_{3}\end{array}$ & $3343 ; 3320$ & 3060 & 1600 & 1648 & ----- & $\begin{array}{l}----- \\
\end{array}$ \\
\hline $5 \mathbf{b}$ & $\begin{array}{c}\mathrm{MoO}_{2}\left(\mathrm{hbp}^{-}\right. \\
\mathrm{SAH}) ; \mathrm{MoC}_{20} \mathrm{H}_{16} \mathrm{~N}_{2} \mathrm{O}_{6}\end{array}$ & $3343 ; 1624$ & ----- & $1595.01 ; 1604.45$ & ----- & 1248 & $943 ; 893$ \\
\hline $6 \mathbf{a}$ & $\mathrm{H}_{2}$ sal-SAH; $\mathrm{C}_{14} \mathrm{H}_{12} \mathrm{~N}_{2} \mathrm{O}_{3}$ & $3290 ; 3200$ & 3050 & 1618 & 1655 & ----- & ----- \\
\hline $6 b$ & $\begin{array}{l}\mathrm{MoO}_{2}(\mathrm{sal}-\mathrm{SAH}) \\
; \mathrm{MoC}_{14} \mathrm{H}_{12} \mathrm{~N}_{2} \mathrm{O}_{6}\end{array}$ & $3290 ; 1624$ & ------- & $1601 ; 1552$ & -------- & 1246 & $891 ; 910$ \\
\hline
\end{tabular}

Table 3 : Electronic Spectral Data of Ligands (1a-6a) and their Dioxomolybdenum Complexes (1b-6b)

\begin{tabular}{|c|c|c|c|c|c|c|c|c|}
\hline $\begin{array}{l}\text { Sr. } \\
\text { No } \\
\text {. }\end{array}$ & $\begin{array}{c}\text { Ligands/ } \\
\text { Complexes }\end{array}$ & $\begin{array}{c}\text { UV-Vis } \\
\operatorname{Anm}\left(\in M^{-}\right. \\
\left.{ }^{1} \mathbf{c m}^{-1}\right)\end{array}$ & $\begin{array}{l}\text { Sr } \\
\dot{N} \\
0\end{array}$ & $\begin{array}{c}\text { Ligands/ } \\
\text { Complexes }\end{array}$ & $\begin{array}{c}\text { UV-Vis } \\
\boldsymbol{\lambda} \\
\mathbf{n m}(\in \mathbf{M} \\
\left.-1 \mathbf{c m}^{-1}\right)\end{array}$ & $\begin{array}{c}\text { Sr } \\
\dot{\mathbf{N}} \\
\mathbf{0}\end{array}$ & $\begin{array}{c}\text { Ligands/ } \\
\text { Complexes }\end{array}$ & $\begin{array}{c}\text { UV-Vis } \\
\underset{\mathbf{n m}(\in \mathbf{M}-}{\left.{ }^{1} \mathbf{c m}^{-1}\right)}\end{array}$ \\
\hline $1 \mathrm{a}$ & $\begin{array}{c}\mathrm{H}_{2} \mathrm{hbp}-\mathrm{NAH} \\
\mathrm{C}_{19} \mathrm{H}_{15} \mathrm{~N}_{3} \mathrm{O}_{2}\end{array}$ & $\begin{array}{c}295 \\
\left(1.7 \times 10^{4}\right) \\
330 \\
\left(1.7 \times 10^{4}\right)\end{array}$ & $\mathbf{3 a}$ & $\begin{array}{c}\mathrm{H}_{2} \mathrm{hbp}-\mathrm{BAH} \\
\mathrm{C}_{20} \mathrm{H}_{16} \mathrm{~N}_{2} \mathrm{O}_{2}\end{array}$ & $\begin{array}{c}300 \\
(1.9 \times 10 \\
4) \\
350 \\
\left(\begin{array}{c}1.9 \times 10 \\
4)\end{array}\right.\end{array}$ & $5 a$ & $\begin{array}{c}\mathrm{H}_{2} \mathrm{hbp}-\mathrm{SAH} \\
\mathrm{C}_{20} \mathrm{H}_{16} \mathrm{~N}_{2} \mathrm{O}_{3}\end{array}$ & $\begin{array}{c}300 \\
\left(1.0 \times 10^{4}\right) \\
360 \\
\left(1.0 \times 10^{4}\right)\end{array}$ \\
\hline 1b & $\begin{array}{c}\mathrm{MoO}_{2}(\mathrm{hbp}- \\
\mathrm{NAH}) \\
\mathrm{MoC}_{21} \mathrm{H}_{19} \mathrm{~N}_{3} \\
\mathrm{O}_{5}\end{array}$ & $\begin{array}{c}405 \\
\left(7.5 \times 10^{3}\right)\end{array}$ & $\mathbf{3 b}$ & $\begin{array}{c}\mathrm{MoO}_{2}(\mathrm{hbp}- \\
\mathrm{BAH}) \\
\mathrm{MoC}_{22} \mathrm{H}_{20} \mathrm{~N}_{2} \\
\mathrm{O}_{5}\end{array}$ & $\begin{array}{c}405 \\
(1.9 \times 10 \\
4)\end{array}$ & $5 b$ & $\begin{array}{c}\mathrm{MoO}_{2}(\mathrm{hbp}- \\
\mathrm{SAH}) \\
\mathrm{MoC}_{20} \mathrm{H}_{16} \mathrm{~N}_{2} \\
\mathrm{O}_{6}\end{array}$ & $\begin{array}{c}405(1.0 \times 1 \\
\left.0^{4}\right)\end{array}$ \\
\hline $2 a$ & $\begin{array}{l}\mathrm{H}_{2} \text { sal-NAH } \\
\mathrm{C}_{13} \mathrm{H}_{11} \mathrm{~N}_{3} \mathrm{O}_{2}\end{array}$ & $\begin{array}{c}295 \\
\left(2.1 \times 10^{4}\right) \\
330 \\
\left(2.0 \times 10^{4}\right)\end{array}$ & $4 a$ & $\begin{array}{l}\mathrm{H}_{2} \text { sal-BAH } \\
\mathrm{C}_{14} \mathrm{H}_{12} \mathrm{~N}_{2} \mathrm{O}_{2}\end{array}$ & $\begin{array}{c}280 \\
(1.6 \times 10 \\
4) \\
330 \\
(2.9 \times 10 \\
4)\end{array}$ & $6 a$ & $\begin{array}{l}\mathrm{H}_{2} \text { sal-SAH } \\
\mathrm{C}_{14} \mathrm{H}_{12} \mathrm{~N}_{2} \mathrm{O}_{3}\end{array}$ & $\begin{array}{c}297 \\
\left(1.7 \times 10^{4}\right) \\
335 \\
\left(2.2 \times 10^{4}\right)\end{array}$ \\
\hline 2b & $\begin{array}{c}\mathrm{MoO}_{2} \text { (sal- } \\
\mathrm{NAH}) \\
\mathrm{MoC}_{15} \mathrm{H}_{15} \mathrm{~N}_{3} \\
\mathrm{O}_{5}\end{array}$ & $\begin{array}{c}410(0.3 \times 1 \\
\left.0^{4}\right)\end{array}$ & 4b & $\begin{array}{c}\mathrm{MoO}_{2}(\mathrm{sal}- \\
\mathrm{BAH}) \\
\mathrm{MoC}_{16} \mathrm{H}_{16} \mathrm{~N}_{2} \\
\mathrm{O}_{5} \\
\end{array}$ & $\begin{array}{c}410 \\
(0.3 \times 10 \\
4)\end{array}$ & $6 b$ & $\begin{array}{c}\mathrm{MoO}_{2}(\mathrm{sal}- \\
\mathrm{SAH} \\
\mathrm{MoC}_{14} \mathrm{H}_{12} \mathrm{~N}_{2} \\
\mathrm{O}_{6}\end{array}$ & $\begin{array}{c}410 \\
\left(1.95 \times 10^{4}\right)\end{array}$ \\
\hline
\end{tabular}


Table $4:{ }^{1} \mathrm{H}$ NMR Spectral Data of Ligands (1a-6a) and Complexes (1b-6b)

\begin{tabular}{|c|c|c|}
\hline Sr.No. & $\begin{array}{l}\text { Ligands/ } \\
\text { Complexes }\end{array}$ & б, ppm \\
\hline $\mathbf{1 a}$ & $\begin{array}{c}\mathrm{H}_{2} \mathrm{hbp}- \\
\mathrm{NAH} ; \mathrm{C}_{19} \mathrm{H}_{15} \mathrm{~N}_{3} \mathrm{O}_{2}\end{array}$ & 11.70(s, OH,1H); 12.09(s,NH,1H); 6.97-8.02m, ArH, 13H). \\
\hline $\mathbf{1 b}$ & $\begin{array}{c}\mathrm{MoO}_{2}(\mathrm{hbp}- \\
\mathrm{NAH}) ; \mathrm{MoC}_{21} \mathrm{H}_{19} \mathrm{~N}_{3} \mathrm{O}_{5}\end{array}$ & $\begin{array}{l}11.70(\mathrm{~s}, \mathrm{OH}, 1 \mathrm{H}) ; 1.28-1.42\left(\mathrm{q},-\mathrm{CH}_{3}, 3 \mathrm{H}\right) ; 2.12- \\
\left.2.19\left(\mathrm{t}, \mathrm{CH}_{2}, 2 \mathrm{H}\right) ; 6.97-8.02 \mathrm{~m}, \mathrm{ArH}, 13 \mathrm{H}\right) .\end{array}$ \\
\hline $2 \mathbf{a}$ & $\begin{array}{c}\mathrm{H}_{2} \mathrm{sal}_{-} \\
\mathrm{NAH} ; \mathrm{C}_{13} \mathrm{H}_{11} \mathrm{~N}_{3} \mathrm{O}_{2} \\
\end{array}$ & $\begin{array}{l}11.43(\mathrm{~s}, \mathrm{OH}, 1 \mathrm{H}) ; 12.20(\mathrm{~s}, \mathrm{NH}, 1 \mathrm{H}) ; 8.3(\mathrm{~s}, \mathrm{CH}, 1 \mathrm{H}) ; 6.87- \\
8.75(\mathrm{~m}, \mathrm{ArH}, 8 \mathrm{H}) .\end{array}$ \\
\hline $\mathbf{2 b}$ & $\begin{array}{c}\mathrm{MoO}_{2}\left(\mathrm{sal}_{-}\right. \\
\mathrm{NAH}) ; \mathrm{MoC}_{15} \mathrm{H}_{15} \mathrm{~N}_{3} \mathrm{O}_{5}\end{array}$ & $\begin{array}{l}12.91(\mathrm{~s}, \mathrm{OH}, 1 \mathrm{H}) ; 8.34(\mathrm{~s}, \mathrm{CH}, 1 \mathrm{H}) 1.06-1.17\left(\mathrm{q},-\mathrm{CH}_{3}, 3 \mathrm{H}\right) ; \\
3.16-3.31\left(\mathrm{t}, \mathrm{CH}_{2}, 2 \mathrm{H}\right) ; 6.87-8.75(\mathrm{~m}, \mathrm{ArH}, 8 \mathrm{H}) .\end{array}$ \\
\hline $3 a$ & $\begin{array}{l}\mathrm{H}_{2} \mathrm{hbp}-\mathrm{BAH} \\
; \mathrm{C}_{20} \mathrm{H}_{16} \mathrm{~N}_{2} \mathrm{O}_{2}\end{array}$ & $10.92(\mathrm{~s}, \mathrm{OH}, 1 \mathrm{H}) ; 13.1(\mathrm{~s}, \mathrm{NH}, 1 \mathrm{H}) ; 7.08-8.03(\mathrm{~m}, \mathrm{ArH}, 14 \mathrm{H})$. \\
\hline $\mathbf{3 b}$ & $\begin{array}{c}\mathrm{MoO}_{2}(\mathrm{hbp}- \\
\mathrm{BAH}) ; \mathrm{MoC}_{22} \mathrm{H}_{20} \mathrm{~N}_{2} \mathrm{O}_{5}\end{array}$ & $\begin{array}{l}11.1(\mathrm{~s}, \mathrm{OH}, 1 \mathrm{H}) ; 1.21-1.29\left(\mathrm{q},-\mathrm{CH}_{3}, 3 \mathrm{H}\right) ; 2.56-2.57(\mathrm{t},- \\
\left.\mathrm{CH}_{2}, 3 \mathrm{H}\right) ; 7.01-8.05(\mathrm{~m}, \mathrm{ArH}, 14 \mathrm{H}) .\end{array}$ \\
\hline $4 a$ & $\begin{array}{c}\mathrm{H}_{2} \mathrm{Sal}_{-} \\
\mathrm{BAH} ; \mathrm{C}_{14} \mathrm{H}_{12} \mathrm{~N}_{2} \mathrm{O}_{2}\end{array}$ & $\begin{array}{l}11.58(\mathrm{~s}, \mathrm{OH}, 1 \mathrm{H}) ; 11.82(\mathrm{~s}, \mathrm{NH}, 1 \mathrm{H}) ; 8.53(\mathrm{~s}, \mathrm{CH}, 1 \mathrm{H}) ; 6.86- \\
8.5(\mathrm{~m}, \mathrm{ArH}, 9 \mathrm{H}) .\end{array}$ \\
\hline 4b & $\begin{array}{l}\mathrm{MoO}_{2}(\mathrm{sal}-\mathrm{BAH}) \\
; \mathrm{MoC}_{16} \mathrm{H}_{16} \mathrm{~N}_{2} \mathrm{O}_{5}\end{array}$ & $\begin{array}{l}11.58(\mathrm{~s}, \mathrm{OH}, 1 \mathrm{H}) ; 8.53(\mathrm{~s}, \mathrm{CH}, 1 \mathrm{H}) ; 1.37-1.41(\mathrm{q},- \\
\left.\mathrm{CH}_{3}, 3 \mathrm{H}\right) ; 3.38-3.44\left(\mathrm{t},-\mathrm{CH}_{2}, 3 \mathrm{H}\right) ; 6.94-8.12(\mathrm{~m}, \mathrm{ArH}, 9 \mathrm{H}) .\end{array}$ \\
\hline $5 a$ & $\begin{array}{c}\mathrm{H}_{2} \mathrm{hbp}- \\
\mathrm{SAH} ; \mathrm{C}_{20} \mathrm{H}_{16} \mathrm{~N}_{2} \mathrm{O}_{3}\end{array}$ & $11.52(\mathrm{~s}, \mathrm{OH}, 2 \mathrm{H}) ; 10.4(\mathrm{~s}, \mathrm{NH}, 1 \mathrm{H}) ; 7.54-8.28(\mathrm{~m}, \mathrm{ArH}, 13 \mathrm{H})$ \\
\hline $\mathbf{5 b}$ & $\begin{array}{c}\mathrm{MoO}_{2}\left(\mathrm{hbp}_{-}\right. \\
\mathrm{SAH}) ; \mathrm{MoC}_{20} \mathrm{H}_{16} \mathrm{~N}_{2} \mathrm{O}_{6}\end{array}$ & $\begin{array}{l}11.5(\mathrm{~s}, \mathrm{OH}, 1 \mathrm{H}) ; 1.4-1.64\left(\mathrm{q}, \mathrm{CH}_{3}, 3 \mathrm{H}\right) ; 2,81-2.98\left(\mathrm{t}, \mathrm{CH}_{2}, 2 \mathrm{H}\right) \\
7.4-7.81(\mathrm{~m}, \mathrm{ArH}, 13 \mathrm{H}) .\end{array}$ \\
\hline 6a & $\begin{array}{c}\mathrm{H}_{2} \mathrm{Sal}_{-} \\
\mathrm{SAH} ; \mathrm{C}_{14} \mathrm{H}_{12} \mathrm{~N}_{2} \mathrm{O}_{3} \\
\end{array}$ & $\begin{array}{ll}11.97(\mathrm{~s}, \mathrm{OH}, 2 \mathrm{H}) ; & 11.40(\mathrm{~s}, \mathrm{NH}, 1 \mathrm{H}) ; 8.6(\mathrm{~s}, \mathrm{CH}, 1 \mathrm{H}) \\
6.8-7.93(\mathrm{~m}, \mathrm{ArH}, 8 \mathrm{H}) & \\
\end{array}$ \\
\hline $6 b$ & $\begin{array}{l}\mathrm{MoO}_{2}(\mathrm{sal}-\mathrm{SAH}) \\
\mathrm{MoC}_{14} \mathrm{H}_{12} \mathrm{~N}_{2} \mathrm{O}_{6}\end{array}$ & $11.31(\mathrm{~s}, \mathrm{OH}, 1 \mathrm{H}) ; 8.68(\mathrm{~s}, \mathrm{CH}, 1 \mathrm{H}) ; 6.90-8.68(\mathrm{~m}, \mathrm{ArH}, 8 \mathrm{H})$ \\
\hline
\end{tabular}

Table 5: Thermal Decomposition Data of complexes (1b-6b)

\begin{tabular}{|c|c|c|c|c|c|c|c|c|c|c|c|c|c|}
\hline \multirow{3}{*}{$\begin{array}{c}\text { Sr } \\
\text {. } \\
\text { o. }\end{array}$} & \multirow{3}{*}{$\begin{array}{c}\mathbf{T}_{\mathbf{d}} \\
\left({ }^{\circ} \mathbf{C}\right)\end{array}$} & \multicolumn{12}{|c|}{ \% Weight Loss } \\
\hline & & \multicolumn{2}{|c|}{$\begin{array}{c}1 b \\
\mathrm{MoO}_{2}(\mathrm{hbp}- \\
\mathrm{NAH} \\
\mathrm{MoC}_{21} \mathrm{H}_{19} \mathrm{~N} \\
{ }_{3} \mathrm{O}_{5}\end{array}$} & \multicolumn{2}{|c|}{$\begin{array}{c}2 b \\
\mathrm{MoO}_{2} \text { (Sal- } \\
\mathrm{NAH} \\
\mathrm{MoC}_{15} \mathrm{H}_{15} \\
\mathrm{~N}_{3} \mathrm{O}_{5} \\
\end{array}$} & \multicolumn{2}{|c|}{$\begin{array}{c}3 \mathbf{b} \\
\mathrm{MoO}_{2}(\mathbf{h b p}- \\
\mathrm{BAH} \\
\mathrm{MoC}_{22} \mathrm{H}_{20} \\
\mathrm{~N}_{2} \mathrm{O}_{5} \\
\end{array}$} & \multicolumn{2}{|c|}{$\begin{array}{c}4 b \\
\mathrm{MoO}_{2} \text { (sal- } \\
\mathrm{BAH} \\
\mathrm{MoC}_{16} \mathrm{H}_{16} \\
\mathrm{~N}_{2} \mathrm{O}_{5} \\
\end{array}$} & \multicolumn{2}{|c|}{$\begin{array}{c}5 b \\
\mathrm{MoO}_{2}(\mathbf{h b p}- \\
\mathrm{SAH} \\
\mathrm{MoC}_{20} \mathrm{H}_{16} \\
\mathrm{~N}_{2} \mathrm{O}_{6}\end{array}$} & \multicolumn{2}{|c|}{$\begin{array}{c}6 b \\
\mathrm{MoO}_{2} \text { (sal- } \\
\text { SAH) } \\
\mathrm{MoC}_{14} \mathrm{H}_{12} \\
\mathrm{~N}_{2} \mathrm{O}_{6}\end{array}$} \\
\hline & & $\begin{array}{c}\text { Cal } \\
\text { c. }\end{array}$ & Obs & $\begin{array}{c}\text { Cal } \\
\text { c. }\end{array}$ & Obs & $\begin{array}{c}\text { Cal } \\
\text { c. }\end{array}$ & Obs & $\begin{array}{c}\text { Cal } \\
\text { c. }\end{array}$ & Obs & $\begin{array}{l}\text { Cal } \\
\text { c. }\end{array}$ & Obs & $\begin{array}{c}\text { Cal } \\
\text { c. }\end{array}$ & Obs \\
\hline 1 & $\begin{array}{c}80- \\
102 \\
{ }^{\circ} \mathrm{C}\end{array}$ & $\begin{array}{c}9.4 \\
0\end{array}$ & $\begin{array}{c}9.2 \\
5\end{array}$ & 9.40 & 9.25 & 9.42 & 8.73 & $\begin{array}{c}11.1 \\
6\end{array}$ & $\begin{array}{c}10.8 \\
8\end{array}$ & 3.70 & 3.52 & 4.50 & 4.20 \\
\hline 2 & $\begin{array}{c}296 \\
- \\
420 \\
{ }^{\circ} \mathrm{C}\end{array}$ & $\begin{array}{l}44 . \\
02\end{array}$ & $\begin{array}{l}43 . \\
82\end{array}$ & $\begin{array}{c}44.0 \\
2\end{array}$ & $\begin{array}{c}43.8 \\
2\end{array}$ & $\begin{array}{c}44.1 \\
2\end{array}$ & $\begin{array}{c}44.4 \\
5\end{array}$ & $\begin{array}{c}32.5 \\
0\end{array}$ & $\begin{array}{c}33.2 \\
1\end{array}$ & $\begin{array}{c}42.5 \\
8\end{array}$ & 42 & $\begin{array}{c}31.1 \\
5\end{array}$ & $\begin{array}{c}32.1 \\
5\end{array}$ \\
\hline 3 & $\begin{array}{c}420 \\
- \\
770 \\
{ }^{\circ} \mathrm{C}\end{array}$ & $\begin{array}{l}48 . \\
39\end{array}$ & $\begin{array}{l}47 . \\
71\end{array}$ & $\begin{array}{c}48.3 \\
9\end{array}$ & $\begin{array}{c}47.6 \\
2\end{array}$ & $\begin{array}{c}48.1 \\
8\end{array}$ & $\begin{array}{c}48.7 \\
2\end{array}$ & $\begin{array}{c}48.1 \\
8\end{array}$ & $\begin{array}{c}47.4 \\
7\end{array}$ & $\begin{array}{c}51.3 \\
4\end{array}$ & $\begin{array}{c}50.5 \\
0\end{array}$ & $\begin{array}{c}51.3 \\
4\end{array}$ & $\begin{array}{c}52.1 \\
0\end{array}$ \\
\hline
\end{tabular}




\section{$T_{d}$ - Decomposition temperature}

Table 6: X-ray Diffraction Data of representive Complex $\mathrm{MoO}_{2}(\mathrm{hbp}-\mathrm{NAH})$ $\left(\mathrm{C}_{2} \mathrm{H}_{5} \mathrm{OH}\right)(1 \mathrm{~b})$

\begin{tabular}{|c|c|c|c|c|c|c|c|c|}
\hline \multirow{2}{*}{$\mathrm{h}$} & \multirow{2}{*}{$\mathrm{k}$} & \multirow{2}{*}{1} & $2 \theta$ & $2 \theta$ & $2 \theta$ & $\mathrm{d}$ & $\mathrm{d}$ & Intensity \\
\hline & & & (Exp.) & (Calc.) & (Diff.) & (Exp.) & (Calc.) & (Exp.) \\
\hline-1 & 0 & 0 & 11.357 & 11.678 & -0.321 & 7.78530 & 7.57183 & 101.42 \\
\hline 0 & 1 & 1 & 11.796 & 11.774 & 0.022 & 7.49612 & 7.51027 & 99.76 \\
\hline 0 & -1 & 1 & 13.426 & 13.477 & -0.051 & 6.58947 & 6.56468 & 327.81 \\
\hline 1 & 1 & 1 & 13.835 & 14.051 & -0.216 & 6.39554 & 6.29791 & 161.00 \\
\hline 1 & 1 & 1 & 14.456 & 14.051 & 0.405 & 6.12245 & 6.29791 & 87.27 \\
\hline-1 & 0 & 1 & 15.520 & 15.688 & -0.168 & 5.70475 & 5.64406 & 115.84 \\
\hline-1 & 0 & 1 & 15.930 & 15.688 & 0.242 & 5.55902 & 5.64406 & 121.18 \\
\hline 0 & 0 & 2 & 17.767 & 17.603 & 0.164 & 4.98823 & 5.03421 & 163.40 \\
\hline 0 & 2 & 0 & 18.087 & 18.255 & -0.168 & 4.90053 & 4.85590 & 176.50 \\
\hline 0 & 1 & 2 & 18.629 & 18.722 & -0.093 & 4.75936 & 4.73593 & 113.65 \\
\hline 1 & 0 & 2 & 19.548 & 19.581 & -0.033 & 4.53756 & 4.53004 & 185.45 \\
\hline 1 & 0 & 2 & 19.936 & 19.581 & 0.356 & 4.45005 & 4.53004 & 170.05 \\
\hline 0 & -1 & 2 & 20.763 & 20.918 & -0.154 & 4.27456 & 4.24339 & 286.22 \\
\hline 0 & -2 & 1 & 21.225 & 21.335 & -0.110 & 4.18263 & 4.16128 & 238.64 \\
\hline-1 & 0 & 2 & 22.251 & 22.664 & -0.413 & 3.99196 & 3.92019 & 189.83 \\
\hline 1 & 2 & 2 & 23.235 & 23.252 & -0.016 & 3.82513 & 3.82247 & 110.25 \\
\hline-1 & -1 & 2 & 24.305 & 24.402 & -0.097 & 3.65917 & 3.64482 & 115.68 \\
\hline-1 & 2 & 1 & 25.013 & 25.180 & -0.167 & 3.55713 & 3.53390 & 196.93 \\
\hline 2 & 2 & 1 & 25.844 & 25.941 & -0.097 & 3.44459 & 3.43199 & 134.99 \\
\hline 2 & 2 & 0 & 26.482 & 26.522 & -0.041 & 3.36307 & 3.35802 & 111.12 \\
\hline 0 & 3 & 0 & 27.520 & 27.531 & -0.010 & 3.23847 & 3.23727 & 140.91 \\
\hline 2 & 2 & 2 & 28.120 & 28.319 & -0.199 & 3.17075 & 3.14895 & 113.76 \\
\hline 2 & 2 & 2 & 28.492 & 28.319 & 0.173 & 3.13019 & 3.14895 & 122.16 \\
\hline-1 & -2 & 2 & 28.818 & 29.144 & -0.326 & 3.09553 & 3.06163 & 107.83 \\
\hline 1 & 3 & 2 & 29.557 & 29.618 & -0.061 & 3.01976 & 3.01371 & 135.30 \\
\hline-2 & -2 & 1 & 29.856 & 29.904 & -0.048 & 2.99025 & 2.98552 & 123.59 \\
\hline-1 & -3 & 1 & 30.641 & 30.608 & 0.033 & 2.91543 & 2.91850 & 91.81 \\
\hline 2 & 1 & 3 & 31.575 & 31.566 & 0.009 & 2.83124 & 2.83206 & 105.09 \\
\hline-2 & -1 & 2 & 32.229 & 32.237 & -0.008 & 2.77523 & 2.77460 & 81.91 \\
\hline 2 & 0 & 3 & 32.800 & 32.761 & 0.039 & 2.72823 & 2.73140 & 95.55 \\
\hline 2 & 3 & 2 & 33.403 & 33.086 & 0.317 & 2.68036 & 2.70531 & 95.70 \\
\hline 3 & 1 & 1 & 34.118 & 34.093 & 0.025 & 2.62582 & 2.62767 & 94.95 \\
\hline 3 & 1 & 0 & 34.764 & 34.710 & 0.054 & 2.57849 & 2.58234 & 108.33 \\
\hline 3 & 2 & 1 & 35.496 & 35.458 & 0.038 & 2.52698 & 2.52960 & 80.00 \\
\hline-3 & -1 & 1 & 37.714 & 37.576 & 0.138 & 2.38328 & 2.39171 & 101.91 \\
\hline 2 & 1 & 4 & 38.380 & 38.460 & -0.080 & 2.34346 & 2.33875 & 90.36 \\
\hline-1 & 1 & 4 & 40.296 & 40.000 & 0.297 & 2.23632 & 2.25222 & 79.43 \\
\hline
\end{tabular}


Table7: X-ray Diffraction Data of representative Complex $\mathrm{MoO}_{2}$ (sal$\mathrm{NAH})\left(\mathrm{C}_{2} \mathrm{H}_{5} \mathrm{OH}\right)(2 \mathrm{~b})$

\begin{tabular}{|c|c|c|c|c|c|c|c|c|}
\hline $\mathbf{H}$ & \multirow{2}{*}{$\mathbf{k}$} & $\mathbf{1}$ & $\mathbf{2} \theta$ & $\mathbf{2} \theta$ & $\mathbf{2} \theta$ & $\mathbf{d}$ & $\mathbf{d}$ & Intensity \\
\cline { 4 - 9 } & & & $\mathbf{\text { Exp.) }}$ & (Calc.) & (Diff.) & (Exp.) & (Calc.) & (Exp.) \\
\hline 0 & 1 & 1 & 11.522 & 11.679 & -0.157 & 7.67403 & 7.57091 & 61.00 \\
\hline 0 & -1 & 1 & 12.799 & 13.283 & -0.484 & 6.91080 & 6.66017 & 259.21 \\
\hline 1 & 0 & 1 & 13.762 & 13.741 & 0.021 & 6.42940 & 6.43920 & 126.15 \\
\hline 1 & 1 & 1 & 14.893 & 14.615 & 0.278 & 5.94369 & 6.05599 & 78.91 \\
\hline 1 & 1 & 1 & 15.105 & 14.615 & 0.490 & 5.86055 & 6.05599 & 61.47 \\
\hline-1 & 1 & 0 & 16.280 & 16.187 & 0.093 & 5.44021 & 5.47132 & 154.08 \\
\hline 0 & 2 & 0 & 17.972 & 18.027 & -0.055 & 4.93158 & 4.91673 & 72.10 \\
\hline 0 & 2 & 1 & 19.017 & 19.003 & 0.014 & 4.66307 & 4.66647 & 177.85 \\
\hline-1 & -2 & 0 & 19.877 & 19.936 & -0.059 & 4.46306 & 4.45003 & 128.66 \\
\hline-1 & 0 & 2 & 22.251 & 22.642 & -0.391 & 3.99201 & 3.92399 & 89.54 \\
\hline-1 & 2 & 0 & 23.218 & 23.361 & -0.142 & 3.82786 & 3.80489 & 28.93 \\
\hline-1 & -1 & 2 & 24.498 & 24.478 & 0.020 & 3.63072 & 3.63360 & 53.75 \\
\hline 1 & -2 & 1 & 25.012 & 25.147 & -0.135 & 3.55724 & 3.53841 & 71.07 \\
\hline 1 & -2 & 1 & 25.617 & 25.147 & 0.470 & 3.47455 & 3.53841 & 116.41 \\
\hline 0 & 0 & 3 & 26.039 & 26.247 & -0.208 & 3.41923 & 3.39263 & 24.37 \\
\hline 2 & 0 & 2 & 27.728 & 27.685 & 0.043 & 3.21468 & 3.21960 & 45.38 \\
\hline 2 & -1 & 1 & 28.319 & 27.850 & 0.469 & 3.14895 & 3.20092 & 34.50 \\
\hline 1 & -1 & 3 & 30.284 & 30.429 & -0.145 & 2.94897 & 2.93526 & 29.45 \\
\hline 2 & -1 & 2 & 31.196 & 31.111 & 0.085 & 2.86481 & 2.87245 & 23.25 \\
\hline-1 & 3 & 1 & 32.471 & 32.427 & 0.044 & 2.75518 & 2.75880 & 23.01 \\
\hline-2 & -1 & 2 & 32.896 & 32.790 & 0.106 & 2.72051 & 2.72908 & 45.17 \\
\hline 1 & -3 & 1 & 33.271 & 33.351 & -0.080 & 2.69068 & 2.68443 & 56.75 \\
\hline-1 & 2 & 3 & 34.900 & 35.016 & -0.116 & 2.56872 & 2.56049 & 20.86 \\
\hline-2 & 0 & 3 & 38.630 & 38.621 & 0.010 & 2.32884 & 2.32940 & 24.00 \\
\hline 2 & 2 & 4 & 40.290 & 40.184 & 0.105 & 2.23667 & 2.24229 & 24.32 \\
\hline 0 & -3 & 3 & 40.585 & 40.605 & -0.019 & 2.22107 & 2.22006 & 22.07 \\
\hline-1 & 2 & 4 & 42.259 & 42.256 & 0.004 & 2.13688 & 2.13705 & 24.72 \\
\hline 0 & -4 & 2 & 42.765 & 42.810 & -0.045 & 2.11279 & 2.11067 & 25.66 \\
\hline-1 & 4 & 2 & 43.210 & 43.221 & -0.011 & 2.09205 & 2.09154 & 20.23 \\
\hline 1 & 5 & 1 & 45.461 & 44.989 & 0.472 & 1.99355 & 2.01336 & 22.04 \\
\hline
\end{tabular}



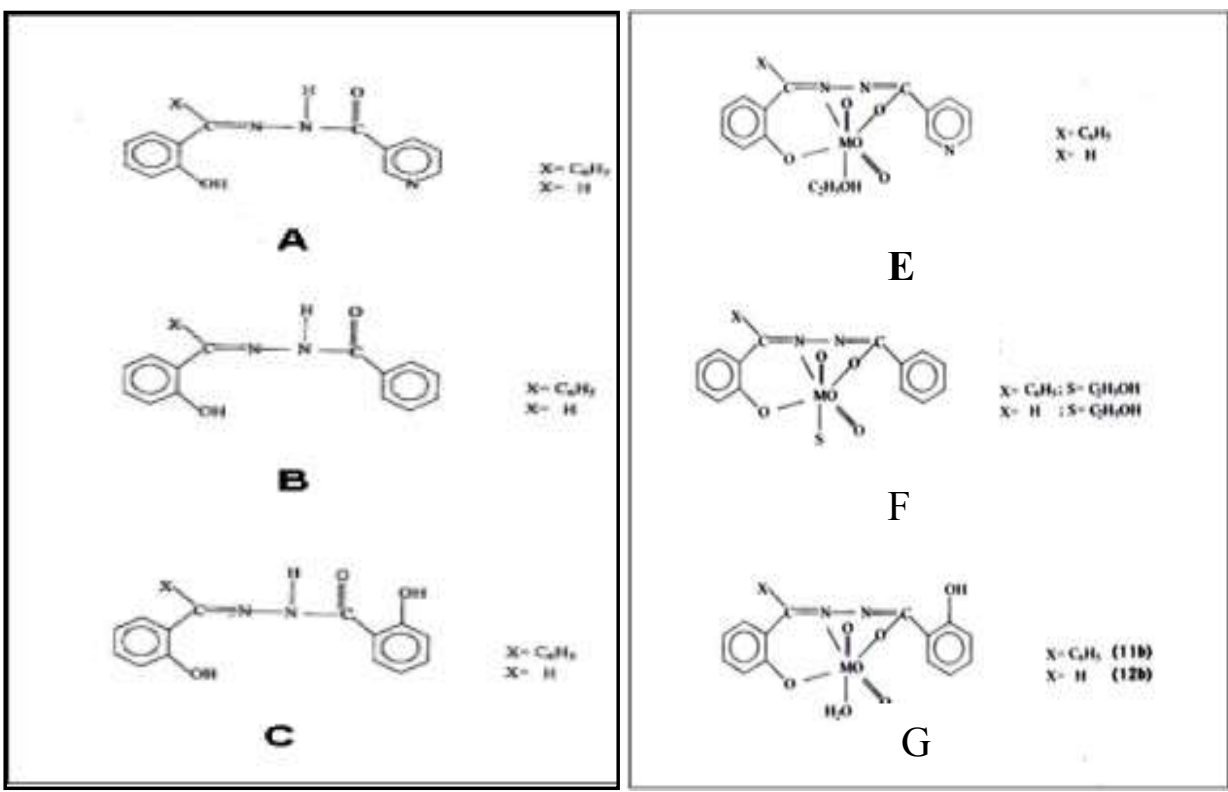

Fig1: A- Proposed structure for 1a and 2a

Fig 2: E- Proposed structure for $1 b$ and $2 b$

B- Proposed structure for $3 a$ and $4 a$ F- Proposed structure for $\mathbf{3 b}$ and $\mathbf{4 b}$

$$
\text { C- Proposed structure for } 5 a \text { and } 6 a
$$

G- Proposed structure for

$5 b$ and $6 b$ 